\title{
Bound states in two-dimensional spin systems near the Ising limit : a quantum finite-lattice study
}

\author{
Sébastien Dusuel, ${ }^{1, *}$ Michael Kamfor, ${ }^{2, \dagger}$ Kai Phillip Schmidt, ${ }^{2,}{ }^{\ddagger}$ Ronny Thomale, ${ }^{2,3,4, \S}$ and Julien Vidal ${ }^{5}$, \\ ${ }^{1}$ Lycée Saint-Louis, 44 Boulevard Saint-Michel, 75006 Paris, France \\ ${ }^{2}$ Lehrstuhl für Theoretische Physik I, Otto-Hahn-Straße 4, TU Dortmund, 44221 Dortmund, Germany \\ ${ }^{3}$ Institut für Theorie der Kondensierten Materie, \\ Universität Karlsruhe, D-76128 Karlsruhe, Germany \\ ${ }^{4}$ Department of Physics, Princeton University, Princeton, New Jersey 08544, USA \\ ${ }^{5}$ Laboratoire de Physique Théorique de la Matière Condensée, CNRS UMR 7600, \\ Université Pierre et Marie Curie, 4 Place Jussieu, 75252 Paris Cedex 05, France
}

\begin{abstract}
We analyze the properties of low-energy bound states in the transverse-field Ising model and in the XXZ model on the square lattice. To this end, we develop an optimized implementation of perturbative continuous unitary transformations. The Ising model is studied in the small-field limit which is found to be a special case of the toric code model in a magnetic field. To analyze the XXZ model, we perform a perturbative expansion about the Ising limit in order to discuss the fate of the elementary magnon excitations when approaching the Heisenberg point.

PACS numbers: 75.10.Jm,05.30.Rt,05.50.+q,03.65.Ge
\end{abstract}

\section{INTRODUCTION}

Properties of strongly correlated quantum matter are usually well described by the many-body ground state and by the first elementary excitation. Multiparticle excitations are often not important because they just constitute an incoherent background. Thus, the study of quantum phase transitions mainly relies on low-energy spectrum analysis, namely energy levels of the ground state and the first excited state ${ }^{1}$. However, in various systems, bound states may arise and play a major role. One of the most famous example are Cooper pairs which lead to superconductivity but other electron systems also display such mechanism (see, for instance, Refs. 2-4). In one-dimensional magnetic systems, well-known examples are the dimerized and frustrated spin chain as well as the two-leg spin ladder ${ }^{5-10}$ which contains bound states made up of triplons. Interestingly, such modes have been experimentally observed in cuprate ladder materials ${ }^{11}$. In two dimensions, the frustrated Shastry-Sutherland model and its experimental realization $\mathrm{SrCu}_{2}\left(\mathrm{BO}_{3}\right)_{2}$ are also known to possess two-triplon bound states ${ }^{12,13}$. Note that more complicated bound states may arise in topologically ordered systems where anyons (semions) can bind to form bosons or fermions as discussed in Ref. 14.

Recently, such binding effects have been studied in the two-dimensional XXZ model ${ }^{15}$ where elementary excitations are dressed magnons. The aim of the present paper is to analyze the spectrum of such magnon bound states in two different spin systems. The first one is the ferromagnetic transverse-field Ising model (TFIM) on the square lattice for which we derive the two-magnon spectrum perturbatively in the small-field limit. The highorder series expansion (order 12) of the corresponding gap allows us to extrapolate its behavior near the critical point where it is found to vanish. We also compute the ratio between the one-magnon gap and this two-magnon gap at the critical point which is approximately 1.8 , in agreement with field-theoretical predictions ${ }^{16,17}$. The second system considered in this study is the XXZ model on the square lattice which, in the isotropic limit (XXX), is the celebrated antiferromagnetic Heisenberg model. As for the TFIM, we focus on the two-magnon bound-states spectrum which is derived up to order 8 near the Ising limit. However, as we shall see, results obtained here differ from those obtained recently by Hamer ${ }^{15}$ since, contrary to his claim, we show using very simple arguments, that there are two distinct branches of twomagnon bound states at low energy. Also, let us underline that the fate of the lowest-energy bound state when approaching the Heisenberg limit cannot be conclusively determined at this order.

From a methodological point of view, several methods to compute high-order series expansion in quantum many-body systems are available (see Refs. 18,19 for a review). Here, we use the perturbative continuous unitary transformations (PCUTs) method ${ }^{20-23}$ which is especially well suited to investigate the manyparticle spectrum and provides a natural quasiparticle (QP) description.

The structure of this paper is the following. In Sec. II, we introduce the two models (TFIM in Sec. II A and XXZ model in Sec. IIB) under consideration. We show that the Ising limit is a good starting point for a perturbation theory in the PCUTs framework and we also give a very simple picture to understand the occurrence of bound states in this limiting case. In Sec. II C, we introduce another description of the spin model in terms of bond degrees of freedom which will be useful to set up the present perturbation theory framework. The end of this Sec. II D is dedicated to symmetry considerations. In Sec. III, we recall several important aspects of PCUTs which are essential to understand the next section. In Sec. IV, we adapt the finite-lattice method (commonly used in statistical mechanics ${ }^{19,24}$ ) to quantum problems, allowing one 


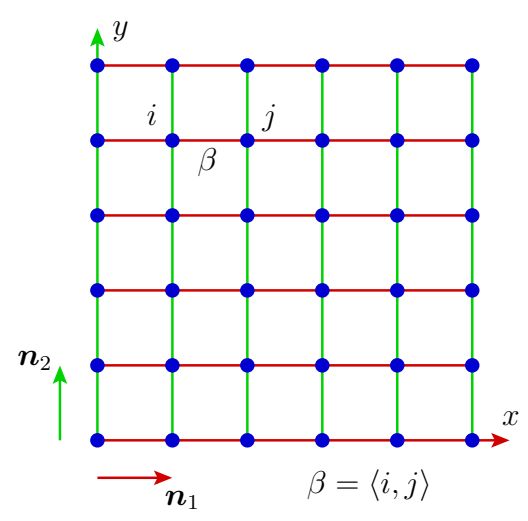

FIG. 1: (Color online) Square lattice on which the models studied in this paper are defined. Translation vectors in the $x$ and $y$ directions are denoted $\boldsymbol{n}_{1}$ and $\boldsymbol{n}_{2}$. The bond between sites $i$ and $j$ is denoted $\langle i, j\rangle$ or by a Greek letter such as $\beta$ to shorten the notation.

to significantly increase the maximum order of the series expansions. Let us stress that readers interested only in results can skip these two sections and switch directly to Secs. V and VI where we discuss the low-energy spectra of the TFIM and XXZ model, respectively. Finally, in Sec. VII, we discuss the spectrum of a new model which naturally emerges when describing the TFIM in terms of bonds. This model may be seen as a special case of the toric code model ${ }^{25}$ in a magnetic field ${ }^{14,26}$ in which flux creation energy cost vanishes. All coefficients used to compute gaps in both models are gathered in Appendices $\mathrm{A}$ and $\mathrm{B}$.

\section{MODELS AND MAPPINGS}

\section{A. Transverse-field Ising model}

Let us first introduce the TFIM on the square lattice whose Hamiltonian reads

$$
H_{\mathrm{TFIM}}=-J \sum_{\langle i, j\rangle} \sigma_{i}^{z} \sigma_{j}^{z}-h \sum_{i} \sigma_{i}^{x}
$$

where the second sum runs over all $i$ of the square lattice and the first sum runs over all bonds $\langle i, j\rangle$ between nearest-neighbor sites (see Fig. 1). The Pauli matrices are denoted by $\sigma^{x}, \sigma^{y}$, and $\sigma^{z}$. Here, we focus on a ferromagnetic Ising coupling $J>0$ and, without loss of generality, we consider $h \geq 0$.

This model whose classical counterpart is the threedimensional Ising model is known to display a secondorder phase transition separating a symmetric (disordered) phase at large field $h$ from a broken (ordered) phase at large coupling $J$. For an infinite field, all spins point in the $+x$ direction, while for an infinite exchange coupling, all spins point either in the $+z$ direction or in the $-z$ direction.

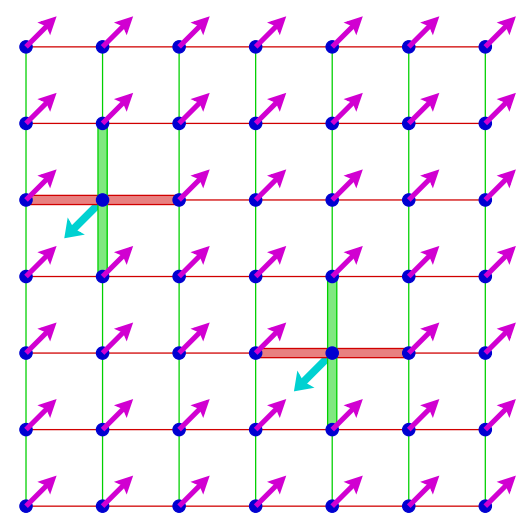

FIG. 2: (Color online) Two magnons (spins pointing left) on top of a ferromagnetic background. Such a state involves eight antiferromagnetic (bold) bonds (four for each magnon), and costs an energy 8 , with respect to Hamiltonian $H_{\mathrm{I}}$.

The ground-state energy as well as the singleexcitation spectrum have been computed in both phases using series expansion ${ }^{19,27,28}$ allowing for a precise determination of the critical point at $J /\left.h\right|_{c}=0.3285(1)$. In the broken phase, the perturbative expansion is done around the Ising limit $(h=0)$. Thus, it is convenient to introduce the following Hamiltonian that will also be the starting point of our study of the Heisenberg model (see Sec. II B)

$$
H_{\mathrm{I}}=-\frac{1}{2} \sum_{\langle i, j\rangle} \sigma_{i}^{z} \sigma_{j}^{z}
$$

Let us consider its ferromagnetic ground state where all spins point in the $+z$ direction. Lowest-energy excitations then consist in static magnons (spin flips) whose energy cost is 4 . The prefactor $1 / 2$ in Eq. (2) defining $H_{\mathrm{I}}$ indeed ensures that any state will have an energy equal to that of the ground state plus the number of antiferromagnetic bonds. Two such "isolated" magnons are shown in Fig. 2.

A two-magnon state will have an energy cost of 8, except if the two magnons are nearest neighbors. Indeed, in such a case, only six bonds have an antiferromagnetic configuration, which results in an energy reduction of $8-6=2$ compared to the situation where magnons are far apart. This proves the existence of bound states in the spectrum, two of which are shown in Fig. 3.

Of course, following the same line of reasoning, one can show that $n$-magnon bound states exist for any $n \geqslant 2$, but we shall restrict our study to the case $n=2$. Let us also notice that in the ordered phase, the perturbation is performed around the field term of Eq. (1), which basically counts spin flips, so that no binding effect is present in this limit. 


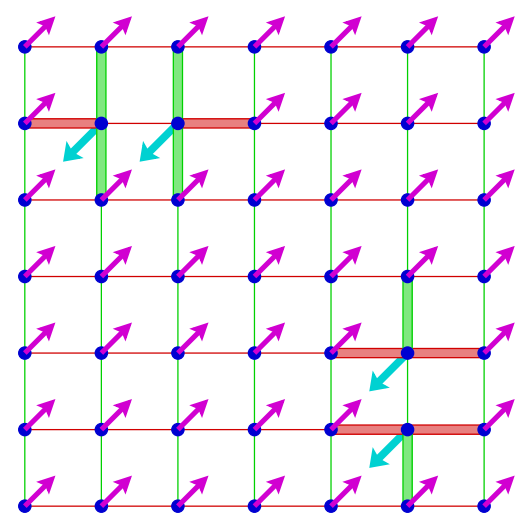

FIG. 3: (Color online) Two examples of two-magnon bound states. Each of them has an energy cost of 6 , with respect to Hamiltonian $H_{\mathrm{I}}$. As in Fig. 2, antiferromagnetic bonds are represented as bold segments.

\section{B. Heisenberg and XXZ models}

Let us now discuss, in the same spirit, the antiferromagnetic Heisenberg model on the square lattice. Its Hamiltonian reads

$$
H_{\mathrm{H}}=J \sum_{\langle i, j\rangle} \boldsymbol{S}_{i} \cdot \boldsymbol{S}_{j},
$$

where $\boldsymbol{S}_{i}=\boldsymbol{\sigma}_{i} / 2$ is the spin operator at site $i$. In the following, we set $J=2$ and we introduce an anisotropy parameter $\lambda$ with the aim of performing series expansion in this parameter, as was done in Refs. 29 and 19,30,31. The Heisenberg Hamiltonian is then the $\lambda=1$ limit of the following XXZ Hamiltonian

$$
\begin{aligned}
H_{\mathrm{XXZ}} & =\frac{1}{2} \sum_{\langle i, j\rangle} \sigma_{i}^{z} \sigma_{j}^{z}+\frac{\lambda}{2} \sum_{\langle i, j\rangle}\left(\sigma_{i}^{x} \sigma_{j}^{x}+\sigma_{i}^{y} \sigma_{j}^{y}\right) \\
& =\frac{1}{2} \sum_{\langle i, j\rangle} \sigma_{i}^{z} \sigma_{j}^{z}+\lambda \sum_{\langle i, j\rangle}\left(\sigma_{i}^{+} \sigma_{j}^{-}+\sigma_{i}^{-} \sigma_{j}^{+}\right),
\end{aligned}
$$

where we have introduced the usual raising and lowering operators $\sigma_{i}^{ \pm}=\frac{1}{2}\left(\sigma_{i}^{x} \pm \mathrm{i} \sigma_{i}^{y}\right)$. The square lattice being bipartite, it is possible to perform a rotation of angle $\pi$ around the $x$ axis on one of the sublattices, namely $\left(\sigma^{x}, \sigma^{y}, \sigma^{z}\right) \rightarrow\left(\sigma^{x},-\sigma^{y},-\sigma^{z}\right)$. This transformation leads, without changing notations for the Pauli operators and making use of $H_{\mathrm{I}}$ defined previously, to

$$
H_{\mathrm{XXZ}}=H_{\mathrm{I}}+\lambda \sum_{\langle i, j\rangle}\left(\sigma_{i}^{+} \sigma_{j}^{+}+\sigma_{i}^{-} \sigma_{j}^{-}\right) .
$$

We thus see that in the limit of a vanishing anisotropy parameter $\lambda, H_{\mathrm{XXZ}}$ reduces to $H_{\mathrm{I}}$ so that the binding effects discussed above for $H_{\text {TFIM }}$ are also at work here. The difference between both models is the perturbation term added to $H_{\mathrm{I}}$.

Let us finally mention that from the point of view of the XXZ model, a second-order quantum phase transition occurs at the Heisenberg point $\lambda=1$, separating a

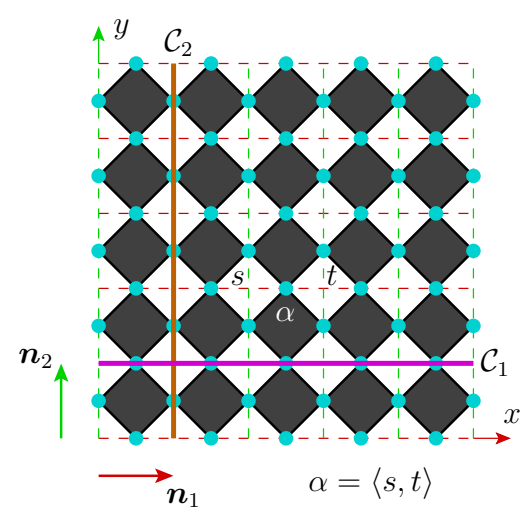

FIG. 4: (Color online) Dots are located in the middle of the bonds of the original square lattice of Fig. 1 (represented with dashed lines). Bond operators of Eq. (7) are defined on these bonds. White plaquettes (centered on vertices of the original lattice) are used to define $\widetilde{A}_{s}$ operators [see Eq. (9)], whereas $\widetilde{B}_{p}$ operators of Eq. (12) are defined on gray plaquettes. The bond that is shared by two white plaquettes such as $s$ and $t$ is denoted by $\langle s, t\rangle$ or by a Greek letter such as $\alpha$. Contours $\mathcal{C}_{1}$ and $\mathcal{C}_{2}$ are used in Eq. (14) to define $\mathbb{Z}_{2}$ conserved quantities for a system with periodic boundary conditions.

gapped phase for $0<\lambda<1$ with a twofold-degenerate ground state with Néel order from a gapless phase with $\mathrm{O}(2)$ symmetry for $\lambda>1$.

\section{Bond description}

As we have already seen in Sec. II A when describing the spectrum of $H_{\mathrm{I}}$, the energy cost of any multimagnon state is given by the number of antiferromagnetic bonds of the state's spin configuration. It is thus natural, though not mandatory, to introduce effective spin variables living on the bonds (see Fig. 4) as follows:

$$
\tilde{\sigma}_{\beta}^{z}=\tilde{\sigma}_{\langle i, j\rangle}^{z}=\sigma_{i}^{z} \sigma_{j}^{z}
$$

To make notations light, we denote bonds $\langle i, j\rangle$ between two sites $i$ and $j$ with Greek letters, such as $\beta$ in the above equation. A value +1 or -1 of $\widetilde{\sigma}_{\beta}^{z}$ is then associated to, respectively, a ferromagnetic or antiferromagnetic configuration of bond $\beta$. Within such a description, Hamiltonian (2) becomes a pure field term

$$
\widetilde{H}_{\mathrm{I}}=-\frac{1}{2} \sum_{\beta} \tilde{\sigma}_{\beta}^{z} .
$$

We can now give another form of the field term of $H_{\mathrm{TFIM}}$. Since $\sigma_{i}^{x}$ flips the spin at site $i$, it flips the four bonds sharing site $i$. Let us denote by $s(i)$ the set of these four bonds (the notation $s$ referring to stars or vertices of the original lattice). Furthermore, we introduce

$$
\widetilde{A}_{s}=\prod_{\beta \in s(i)} \tilde{\sigma}_{\beta}^{x}
$$


which is the product of the four operators flipping bonds connected to site $i$. One can alternatively say that the $\widetilde{A}_{s}$ are defined on the white plaquettes of Fig. 4. Then, the TFIM Hamiltonian reads

$$
\widetilde{H}_{\mathrm{TFIM}}=-\frac{1}{2} \sum_{\beta} \widetilde{\sigma}_{\beta}^{z}-h \sum_{s} \widetilde{A}_{s} .
$$

In the same vein, we can rewrite the XX part of the XXZ Hamiltonian (4). From expression (6), it is clear that this XX term flips two adjacent spins, if these two spins are in a ferromagnetic configuration while it annihilates antiferromagnetic bonds. We recall that in Eq. (6), the sublattice rotation was already used, so that a ferromagnetic configuration corresponds to an original antiferromagnetic configuration in Eqs. (4) and (5). As a consequence, the XX term flips the six bonds around a ferromagnetic bond, and one can write

$$
\widetilde{H}_{\mathrm{XXZ}}=-\frac{1}{2} \sum_{\beta} \tilde{\sigma}_{\beta}^{z}+\lambda \sum_{\langle s, t\rangle} \widetilde{A}_{s} \widetilde{A}_{t} \frac{1+\tilde{\sigma}_{\alpha}^{z}}{2},
$$

where the second sum runs over nearest-neighbor white plaquettes $s$ and $t$ that share a common bond $\alpha=\langle s, t\rangle$ (see Fig. 4), and involves projectors that annihilate an antiferromagnetic configuration of such bonds.

\section{Symmetries, counting, and relation to toric code}

Up to now, we have not yet discussed one fundamental aspect of the bond description, namely, the counting of states. Indeed, if we assume that the original lattice contains $N$ spins half $\boldsymbol{\sigma}$, the mapping brings us to a description with $2 N$ spins half $\widetilde{\sigma}$ since the number of bonds is twice the number of sites (assuming periodic boundary conditions). Thus, it seems that Hilbert spaces of the $\widetilde{H}$ Hamiltonians (8), (10), and (11) are too large, and involve "unphysical" states.

However, looking at these Hamiltonians a bit closer, it is clear that the following operators are conserved

$$
\widetilde{B}_{p}=\prod_{\beta \in p} \widetilde{\sigma}_{\beta}^{z}
$$

In this definition, the product is performed over all bonds belonging to $p$, which can be any of the gray plaquettes shown in Fig. 4 (they are also plaquettes in the original lattice shown in Fig. 1). Among the $N \mathbb{Z}_{2}$ operators that can be defined this way, only $N-1$ can be set independently to \pm 1 , because of the constraint $\prod_{p} \widetilde{B}_{p}=1$ (with periodic boundary conditions).

With such notations, Hamiltonian (10) is nothing but the toric code Hamiltonian ${ }^{25}$ in a magnetic field (we keep using the $\sim$ notations)

$$
\widetilde{H}_{\mathrm{TC}}=-J_{\mathrm{s}} \sum_{s} \widetilde{A}_{s}-J_{\mathrm{p}} \sum_{p} \widetilde{B}_{p}-h_{z} \sum_{\beta} \widetilde{\sigma}_{\beta}^{z},
$$

with $J_{\mathrm{s}}=h, J_{\mathrm{p}}=0$ and $h_{z}=J=1 / 2$. Although the original toric code (with nonvanishing $J_{s}$ and $J_{p}$ ) in a field has already been the subject of some works (see Refs. 32, and 26,33,34), we are not aware of any study of this precise model which has also been recently obtained in a related framework ${ }^{35}$. For this reason, we will discuss some of its properties in Sec. VII.

For periodic boundary conditions, as in the "bare" toric $\operatorname{code}^{25}$ (see also Ref. 36 for a pedagogical description), $\widetilde{B_{p}}$ 's are not the only conserved operators when a magnetic field in the $z$ direction is present. Using contours $\mathcal{C}_{1}$ and $\mathcal{C}_{2}$ depicted in Fig. 4 , one can indeed define the following operators

$$
\widetilde{C}_{1}=\prod_{\beta \in \mathcal{C}_{1}} \tilde{\sigma}_{\beta}^{z}, \quad \text { and } \quad \widetilde{C}_{2}=\prod_{\beta \in \mathcal{C}_{2}} \tilde{\sigma}_{\beta}^{z} .
$$

These are also $\mathbb{Z}_{2}$ conserved quantities, which can be set to \pm 1 independently of the values of the $\widetilde{B}_{p}$ 's.

All in all, we have $(N-1)+2=N+1$ conserved and independent $\mathbb{Z}_{2}$ quantities. Furthermore, to recover the "physical" subspace and the physics of the TFIM or the Heisenberg model, one should set all of them to +1 . This reduces the Hilbert space dimension from $2^{2 N}$ to $2^{2 N-(N+1)}=2^{N-1}$, that is in fact less than the original Hilbert space dimension. However, the reason for this obvious : our description in terms of bond variables is done with respect to one of the two degenerate ferromagnetic ground states. As a consequence, such a description can only be valid in the broken phase.

\section{PERTURBATIVE CONTINUOUS UNITARY TRANSFORMATIONS}

The aim of this section is to equip the reader with the basic knowledge about PCUTs necessary for the understanding of the next section. For concreteness, we work out an example in detail, rather than focusing on a general framework.

\section{A. Basic ideas of continuous unitary transformations}

The CUTs method as known in the condensedmatter theory community originates from the work of Wegner ${ }^{20,37,38}$. A pedagogical introduction to this technique can be found in Refs. 39 and 40. The aim of this technique is to diagonalize or, more modestly, to block diagonalize a given Hamiltonian $H$ thanks to a unitary transformation. The latter is not performed in a single step but rather in a continuous way (whence the name of the method) as

$$
H(l)=U^{\dagger}(l) H U(l)
$$

where $l$ is a running parameter such that $H=H(l=0)$ and $H_{\text {eff }}=H(l=\infty)$ is an effective (block-) diagonal 
Hamiltonian. This equation can be cast into a differential (flow) commutator equation ${ }^{20}$

$$
\partial_{l} H(l)=[\eta(l), H(l)],
$$

where $\eta(l)=\partial_{l} U^{\dagger}(l) U(l)$ is the anti-Hermitian generator associated to the unitary transformation $U(l)$.

\section{B. Quasi-particle conserving generator}

The next task is to find the appropriate generator which, from the local knowledge of $H(l)$, leads to the desired form of the effective Hamiltonian. We shall only discuss the QP conserving generator ${ }^{22}$ that will be used in the sequel. We furthermore focus on a specific example for which the Hamiltonian can be written

$$
H=Q+\sum_{n=-n_{\max }}^{+n_{\max }} T_{n}
$$

For concreteness, in the following we consider the case where $n \in\{0, \pm 2, \pm 4\}$ which is relevant for the TFIM. In this equation, $Q$ is the Hermitian operator which counts the number of QPs (so its spectrum is contained in $\mathbb{N}$ ), and the $T_{n}$ 's are operators that change the QP number by the amount $n$, so that $\left[Q, T_{n}\right]=n T_{n}$. The hermiticity of the Hamiltonian requires that $T_{n}^{\dagger}=T_{-n}$. The $\mathrm{QP}$ conserving generator is designed to bring the Hamiltonian to an effective form that conserves the number of QPs : $\left[Q, H_{\text {eff }}\right]=0$. Said differently, under the CUTs, all terms $T_{n}$ will be flowing (but not $Q$ which is isolated from all other terms), thus becoming $T_{n}(l)$, and one wishes to reach a situation where $T_{n}(l=\infty)=0$ for all $n \neq 0$. This can be achieved ${ }^{22,39,40}$ by choosing

$$
\eta(l)=T_{+2}(l)-T_{-2}(l)+T_{+4}(l)-T_{-4}(l) .
$$

With this choice of generator, the flow Eq. (16) can be written

$$
\begin{aligned}
\partial_{l} T_{0}(l)= & 2\left[T_{+2}(l), T_{-2}(l)\right]+2\left[T_{+4}(l), T_{-4}(l)\right], \\
\partial_{l} T_{+2}(l)= & -2 T_{+2}(l)+\left[T_{+2}(l), T_{0}(l)\right] \\
& +2\left[T_{+4}(l), T_{-2}(l)\right] \\
\partial_{l} T_{+4}(l)= & -4 T_{+4}(l)+\left[T_{+4}(l), T_{0}(l)\right] .
\end{aligned}
$$

We have not written the flow equations for $T_{-2}(l)$ and $T_{-4}(l)$ since the Hamiltonian remains Hermitian under a unitary transformation. Let us emphasize that no new term appears during the flow, thanks to the choice of the generator (e. g., no term creating six particles appears since $\left.\left[T_{+2}(l), T_{+4}(l)\right]+\left[T_{+4}(l), T_{+2}(l)\right]=0\right)$. Linear terms in the right-hand side of Eq. (19) ensure that $T_{n \neq 0}(l=\infty)=0$ so that $\left[H_{\mathrm{eff}}, Q\right]=0$.

\section{Perturbative commutator expansion of the flow equation}

It remains to solve these flow equations which is still a challenging task since the $T_{n}(l)$ terms contain an infinite number of operators. The easiest way of doing so is to perform a perturbative expansion of the flow equations, assuming that all $T_{n}$ terms in $H$ [see Eq. (17)] are small, and of the same order of magnitude. Such an expansion was first performed by Stein $^{21}$ for a Hamiltonian without $T_{ \pm 4}$ terms, and extended to Hamiltonians with terms changing $Q$ by any amount by Knetter and Uhrig ${ }^{22}$ who also provided a description in terms of QP. However the formalism used in these papers was not the same as the one presented here, and, in particular, the emphasis was not on getting a commutator expansion, which is our goal in the following.

To this end, the expansion for $T_{n}(l)$ is simply written

$$
T_{n}(l)=\sum_{i=1}^{\infty} T_{n}^{(i)}(l),
$$

where the superscript $(i)$ is the order, in perturbation, of $T_{n}^{(i)}(l)$. The flow equations can then be expanded as

$$
\begin{aligned}
& \partial_{l} T_{0}^{(i)}(l)= \sum_{j=1}^{i-1}\left(2\left[T_{+2}^{(j)}(l), T_{-2}^{(i-j)}(l)\right]\right. \\
&\left.+2\left[T_{+4}^{(j)}(l), T_{-4}^{(i-j)}(l)\right]\right), \\
& \partial_{l} T_{+2}^{(i)}(l)=-2 T_{+2}^{(i)}(l)+\sum_{j=1}^{i-1}\left(\left[T_{+2}^{(j)}(l), T_{0}^{(i-j)}(l)\right]\right. \\
&\left.+2\left[T_{+4}^{(j)}(l), T_{-2}^{(i-j)}(l)\right]\right), \\
& \partial_{l} T_{+4}^{(i)}(l)=-4 T_{+4}^{(i)}(l)+\sum_{j=1}^{i-1}\left[T_{+4}^{(j)}(l), T_{0}^{(i-j)}(l)\right] .
\end{aligned}
$$

These have to be solved with the initial conditions $T_{n}^{(i)}(l=0)=\delta_{i, 1} T_{n}$ and then one can take the limit $l \rightarrow \infty$ to obtain $H_{\text {eff }}$. For the Hamiltonian considered up to now, one obtains, at order 3 , the following commutator expansion

$$
\begin{aligned}
H_{\mathrm{eff}}= & Q+T_{0}+\frac{1}{2}\left[T_{+2}, T_{-2}\right]+\frac{1}{4}\left[T_{+4}, T_{-4}\right] \\
& +\frac{1}{8}\left(\left[\left[T_{+2}, T_{0}\right], T_{-2}\right]+\left[T_{+2},\left[T_{0}, T_{-2}\right]\right]\right) \\
& +\frac{1}{8}\left(\left[\left[T_{+4}, T_{-2}\right], T_{-2}\right]+\left[T_{+2},\left[T_{+2}, T_{-4}\right]\right]\right) \\
& +\frac{1}{32}\left(\left[\left[T_{+4}, T_{0}\right], T_{-4}\right]+\left[T_{+4},\left[T_{0}, T_{-4}\right]\right]\right) .
\end{aligned}
$$

We have computed such expansions for Hamiltonians of the form given in Eq. (17) to various orders given in Table I. In this table, we also give the total number of nonzero coefficients one obtains once the commutators in the effective Hamiltonian have been expanded in polynomials in $T$ operators. Let us note that for the Hamiltonian we have been considering up to now, with $n \in\{-4,-2,0,2,4\}$, the expansion can be obtained from the one of $n_{\max }=2$ (i. e., $\left.n \in\{-2,-1,0,1,2\}\right)$ by a proper rescaling of the corresponding coefficients. 
Let us also mention that all coefficients are given by rational numbers and are valid for arbitrary system size, including the thermodynamical limit. The very last (but not least) step in the whole computation is to apply this effective Hamiltonian to states with fixed number of QPs and to diagonalize it.

To determine the low-energy QP properties of the TFIM and the XXZ Hamiltonian around the Ising limit, we shall use this PCUTs approach. Indeed, in this limit, both systems can be written in the form (17) by defining the operator

$$
Q=\sum_{\beta} \frac{1-\tilde{\sigma}_{\beta}^{z}}{2}=N+\widetilde{H}_{\mathrm{I}},
$$

which counts the number of antiferromagnetic bonds. The operators $T_{n}$ are then proportional to the perturbation ( $h$ in the TFIM and $\lambda$ in the XXZ model). The index $n$ denotes the change in the number of antiferromagnetic bonds $q$. For the TFIM one has $n \in\{0, \pm 2, \pm 4\}$ and for the XXZ model one gets $n \in\{0, \pm 2, \pm 4, \pm 6\}$. The larger number of $T_{n}$ operators for the XXZ model results in a larger effort since more processes have to be taken into account for a given perturbation order. For instance, for the $2 \mathrm{QP}$ sector (containing bound states), we reached order 12 for the TFIM and order 8 for the XXZ model.

Here, we use the transformed Hamiltonians (10) and (11) defined with bond variables, Eq. (7), but PCUTs can be (and for actual computer implementations are) applied directly to Eqs. (1) and (6). In order to avoid possible confusion, we shall use the notation $0 \mathrm{QP}, 1 \mathrm{QP}$, and $2 \mathrm{QP}$ when referring to 0,1 , and 2 magnons, and 0qp, 1qp, $\ldots, 8 q \mathrm{p}$ when referring to $0,1, \ldots, 8$ antiferromagnetic bonds. The ground state of the effective Hamiltonian is the state without antiferromagnetic bonds $(q=0)$. The one-magnon excitations have $q=4$ and bound states of two nearest-neighbor magnons have $q=6$ antiferromagnetic bonds. Things become much more complicated for configurations with more antiferromagnetic bonds, and we shall not study them here. Let us simply mention that $q=8$ corresponds, in the unperturbed limit, either to two unbound magnons or to three- or four-magnon bound states. However, at finite coupling, we cannot exclude that there also exist two-magnon bound states (different from those discussed above) in this sector.

\begin{tabular}{||c|c|c||}
\hline \hline$n_{\max }$ & Order & Number of coefficients \\
\hline \hline 1 & 18 & 67214380 \\
\hline 2 & 14 & 569842124 \\
\hline 3 & 12 & 924457284 \\
\hline 4 & 10 & 189956506 \\
\hline \hline
\end{tabular}

TABLE I: Maximum order at which we have derived the effective Hamiltonian, for various values of $n_{\max }$ [see Eq. (17) for definitions], as well as the total number of non-zero coefficients needed to express the effective Hamiltonian as a polynomial in $T_{n}$ 's.

\section{LINKED-CLUSTER EXPANSION AND QUANTUM FINITE-LATTICE METHOD}

\section{A. Linked-cluster expansion}

Flow Eq. (21) show that $H_{\text {eff }}$ can be written as a perturbative commutator expansion to any order, as exemplified in Eq. (22) to order 3. This property has dramatic consequences when one considers a Hamiltonian defined on a lattice with local $T_{n}$ operators. By local, we mean that $T_{n}=\sum_{i} T_{n, i}$, where $i$ runs over the lattice sites, with $T_{n, i}$ acting on a finite number of sites neighboring $i$. Indeed, in such a situation, commutators $\left[T_{n, i}, T_{p, j}\right]$ vanish as soon as $i$ and $j$ are sufficiently far apart and are local operators. One could try to implement the calculation of the commutators in a symbolic way but it is usually much easier to apply the effective Hamiltonian (with expanded commutators) to states. For the practical purpose of evaluating the action of one term in $H_{\text {eff }}$, one then only needs to apply $H_{\text {eff }}$ to a finite-size linked cluster of sites. The notion of linked is problem-dependent since it depends on the extension of $T_{n, i}$ operators.

The appearance of a linked-cluster expansion for effective Hamiltonians written as commutators is a longestablished and well-known result (see e. g., Ref. 41), although it seems to have escaped the attention it deserves in more recent publications ${ }^{18,19,23}$.

Let us finally note that the PCUTs method is not the only available one to obtain such a perturbative commutator expansion and one could use the Van Vleck formalism (see e. g., Ref. 42) as well. The main advantage of PCUTs, is that it does not generate terms creating any number of QPs, so that the bookkeeping is not too difficult. One drawback is that one has to solve flow equations instead of performing purely algebraic manipulations.

In the following, we will introduce the two com-

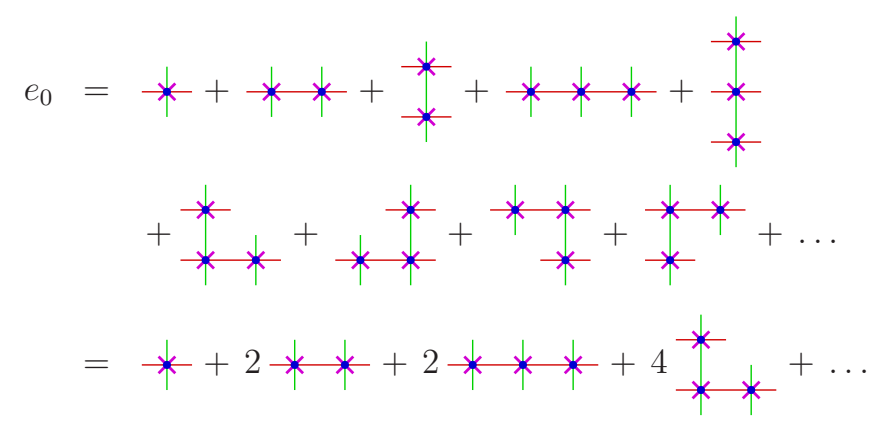

FIG. 5: (Color online) Pictorial representation of the groundstate energy per site as a linked-cluster expansion. A cross on a site means that this site has been acted upon at least twice by a $T_{n}$ operator (two spin flips per site are necessary to preserve the ferromagnetic state). The second line follows from the symmetries of the lattice. All contributions relevant for a computation at order 3 of $e_{0}$ are shown, contrarily to contributions of order 4 (which are all gathered in "..."). 


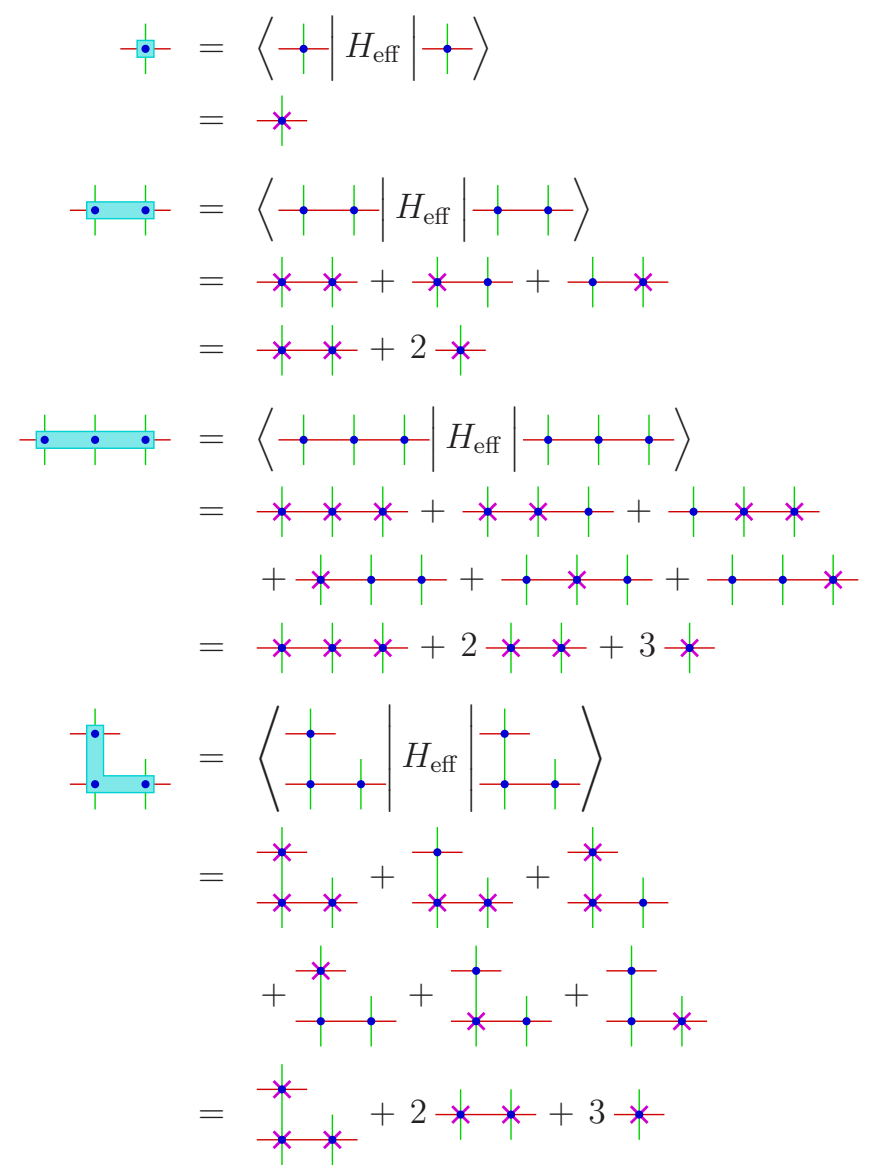

FIG. 6: (Color online) Pictorial representation of the computation of matrix elements of $H_{\text {eff }}$ for various clusters. Crosses have the same meaning as in Fig. 5. Use of symmetry is made to simplify equations and to compute only four graphs (see Fig. 5) but others can be computed as easily.

monly used implementations of the linked-cluster theorem which represent extreme cases. Either a calculation is performed on a large number of minimal clusters or a calculation is done on one very large cluster. Afterwards, a third implementation of the linked-cluster theorem is presented which is a compromise and which we use in the current study.

\section{B. Graph-ology}

With Sec. IV A in mind, it should now be clear that the aim is to compute contributions of the effective Hamiltonian when the latter is allowed to act on states defined on clusters of one site, two sites, etc. To make things more concrete, in what follows, we shall focus on the computation of the ground-state energy per site $e_{0}$ of the TFIM at order 6 in $h$. As only even powers of $h$ will appear in the expansion, we shall for simplicity only refer to the order in $h^{2}$ in this section, so that we will say that we work at order $n$ when computing terms up to order $\left(h^{2}\right)^{n}$. Only

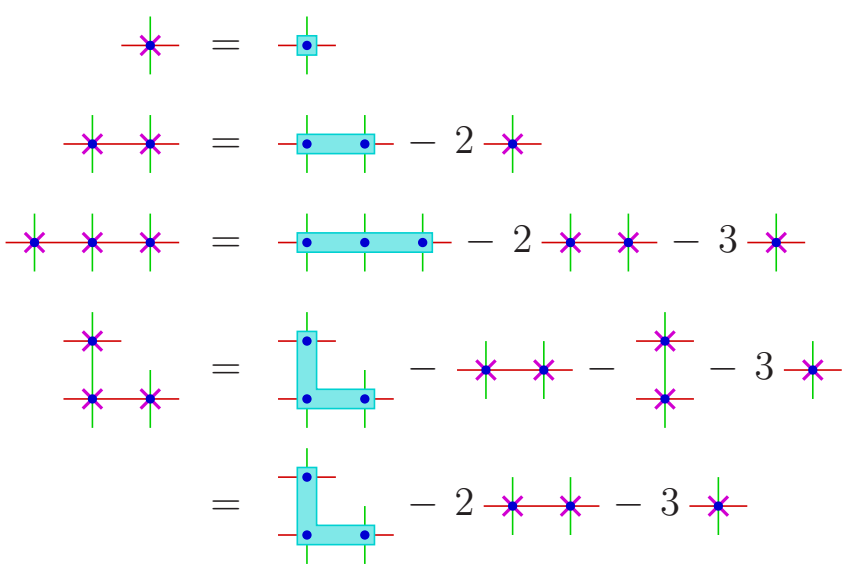

FIG. 7: (Color online) Pictorial representation of the inversion of the relations of Fig. 6, which leads to subcluster subtraction. The last equality is due to symmetries.

the action on the ferromagnetic (0QP) state will be considered here so that we do not draw any arrows to make things clearer. The relevant contributions from different clusters are shown in Fig. 5. Let us mention that the clusters shown in this figure do have contributions at order 4 or more (in which case at least one site is acted upon twice) but clusters not shown (with four crosses or more) do not have contributions at order 3 or less. To obtain the last line of Fig. 5, the symmetries of the lattice have been used. Note that the $T_{n}$ operators can act on at most three sites (each pictogram has at most 3 crosses in Fig. 5) at order 3 and in the 0QP sector, because each $T_{n}$ operator flips the spin of the site on which it acts, so one needs two $T_{n}$ operators per site to start from and end up with a ferromagnetic (0QP) state.

To compute the contributions shown in Fig. 5, it is not very practical to make sure that all sites have been acted upon at least once by a $T_{n}$ operator. It is much easier in actual computations to compute all possible outcomes of the action of $H_{\text {eff }}$ on a given cluster and to subtract contributions of subclusters, as illustrated in Figs. 6 and 7 . Let us note that these subtractions are mandatory in the perturbation theory used in Refs. 18 and 19 which is not based on an expression of $H_{\text {eff }}$ as a series in $T_{n}$ operators. Numerical coefficients appearing in Fig. 7 are simply the number of ways the subclusters (or their symmetric-related ones) can be embedded in a given cluster.

Thus, we are naturally led to enumerate all possible linked clusters ( $i$.e ., also graphs, hence the name of this section) that can be embedded in the square lattice, and apply the subtraction technique to each of the cluster. Though this is the least memory-consuming way to proceed, and though the computational effort required for the computation on one cluster is rather small, it requires to perform a heavy and time-consuming combinatorial work. One indeed has to enumerate all possible linked clusters, and then, for each cluster, one needs to 


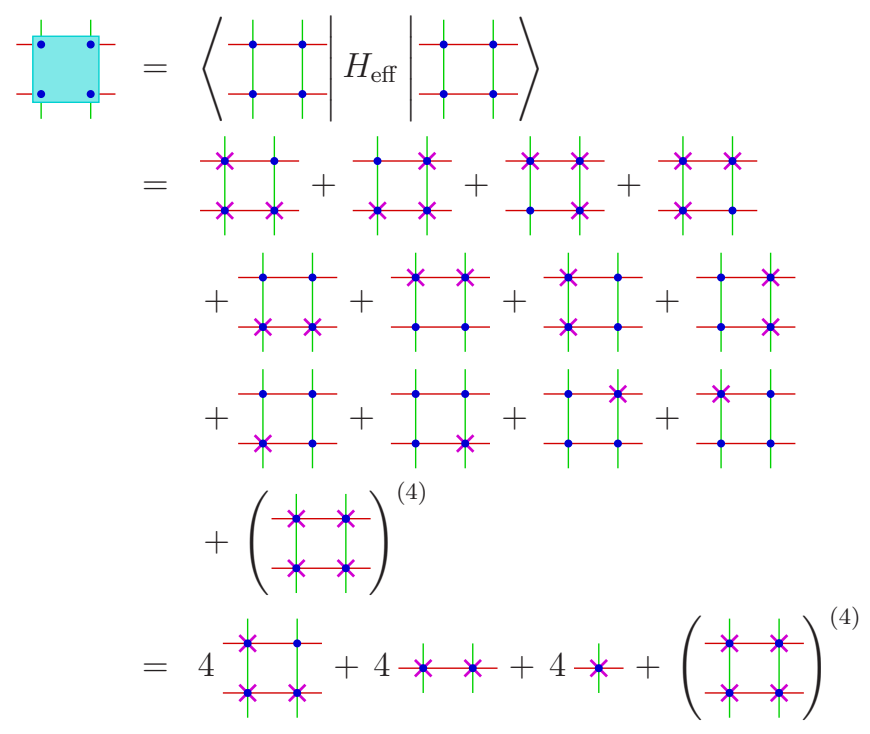

FIG. 8: (Color online) Fourth (and last) matrix element from the $2 \times 2$ rectangular cluster to be considered for a finite-lattice computation at order 3 (apart from the first three of Fig. 6). The term in parentheses involves the action on four sites and only appears at order 4 and beyond (whence the exponent of the parenthesis).

find all its linked subclusters. In order for this technique to be as efficient as possible, one should also ensure that topologically identical clusters are identified (such as the two three-site clusters of Fig. 7). This again reduces the computational effort but makes the combinatorial tasks even harder and more time consuming.

A completely opposite way of performing the calculation is to reduce the combinatorial complexity to zero by computing all contributions in one go, thanks to a cluster with periodic boundary conditions, large enough so that it can accommodate all clusters one is interested in, without any finite-size effect. In this way, applying $H_{\text {eff }}$ to a 0QP state defined on such a cluster one recovers the same state, up to a multiplicative factor being equal to $e_{0}$ times the number of sites of the cluster. However, as the Hilbert-space size quickly grows with the number of sites, this makes all computations greedy in memory, but also in time (because the number of intermediate states generated when applying $H_{\text {eff }}$ can become huge).

\section{Quantum finite-lattice method}

We shall now see how one can work half-way between these two extreme cases. The idea is to generalize Enting's finite-lattice method (see Refs. 19,24,43, and references therein) from the statistical physics setting (where it is used to compute the free energy) to the realm of quantum physics. Essentially, the idea is to use rectangular clusters only and to perform appropriate subtractions. The main advantage of using rectangular clusters is that computing the number of embeddings of rectan-

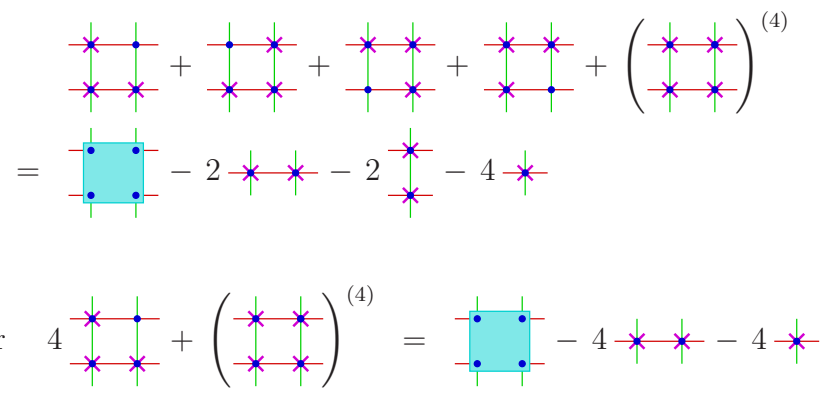

FIG. 9: (Color online) Inversion of the equality of Fig. 8, with the help of the first three lines of Fig. 7, showing how subcluster subtraction appears. The second equality makes use of symmetries, contrarily to the first line.

gular subclusters is trivial. Compared to graph-ology, one gains enormously from the combinatorial side, but of course one has to pay a price : the clusters one uses are larger than those of graph-ology. Enting's finite-lattice method is most useful in low dimensions (namely, two dimensions in statistical mechanics) where the graph sizes do not grow too fast with the order in perturbation but with modifications it can also be efficiently applied to higher dimensions (see Ref. 44 for an application in three dimensions).

Let us first discuss this method in the 0QP sector, where it is almost the same, in the context we are working in, as the original method of statistical mechanics, because the ground state is not infinitely degenerate. In the latter case, our method would provide an effective Hamiltonian in the low-energy subspace which is a major difference compared to a single number (the ground-state energy or the free energy in statistical mechanics). The first three equations of Fig. 6 remain valid since they involve a rectangular cluster but the last equation is now replaced by the new matrix element of Fig. 8 .

This can again be inverted to yield subcluster subtraction and is shown in Fig. 9. From this, one understands that a rectangular cluster yields, after subtraction, the sum of all contributions of the linked subclusters that cannot be embedded in any rectangular subcluster (of the considered cluster). If the cluster has width or height 1 , the sum actually consists in a single term. From these considerations, one can deduce a simple formula. Let us call $D_{s_{x}, s_{y}}$ the expectation value of $H_{\text {eff }}$, computed on a rectangular cluster of size $\left(s_{x}, s_{y}\right)$, so that the first three equations of Fig. 6 and the one of Fig. 8 correspond to $D_{1,1}, D_{2,1}, D_{3,1}$, and $D_{2,2}$. Let us furthermore define (recursively)

$$
\widetilde{D}_{s_{x}, s_{y}}=D_{s_{x}, s_{y}}-\sum_{t_{x}, t_{y}}^{\prime}\left(s_{x}-t_{x}+1\right)\left(s_{y}-t_{y}+1\right) \widetilde{D}_{t_{x}, t_{y}}
$$

where $\sum^{\prime}$ means that the sum is restricted to the set of strict subclusters of sizes $\left(t_{x}, t_{y}\right)$ of the cluster of size $\left(s_{x}, s_{y}\right)$, namely, satisfying $1 \leqslant t_{x} \leqslant s_{x}$ and $1 \leqslant t_{y} \leqslant s_{y}$ with $\left(t_{x}, t_{y}\right) \neq\left(s_{x}, s_{y}\right)$. As an illustration, the first three 
lines of Figs. 7 and 9 give $\widetilde{D}_{1,1}, \widetilde{D}_{2,1}, \widetilde{D}_{3,1}$, and $\widetilde{D}_{2,2}$. Then the ground-state energy can be expressed as a sum of $\widetilde{D}_{s_{x}, s_{y}}$ contributions, for a range of $s_{x}$ and $s_{y}$ that is problem dependent (because the $T_{n}$ operators can have different spatial extensions, as is the case for the TFIM and the Heisenberg model). In the case of the TFIM we have been focusing on up to now, one has

$$
e_{0}=e_{0}^{(0)}+\sum_{n} e_{0}^{(n)}
$$

with

$$
e_{0}^{(n \geqslant 1)}=\sum_{1 \leqslant s_{x}+s_{y}-1 \leqslant n} \widetilde{D}_{s_{x}, s_{y}}
$$

and $e_{0}^{(0)}$ is the constant shift of order 0 in the Hamiltonian. Contrary to the TFIM where $T_{n}$ operators act on one site, the $T_{n}$ 's for the Heisenberg model act on two sites so that the sum for $e_{0}^{(n)}$ would be restricted to $1 \leqslant s_{x}+s_{y}-1 \leqslant 2 n$, where $n$ is the order in $\lambda^{2}$.

The very same idea can be applied for any QP sector, provided appropriate subtractions of contributions from sectors with lower number of QPs are performed, as in standard linked-cluster expansions ${ }^{18,19,23}$. In the same spirit as before, let us give a pictorial explanation based on the TFIM, at order 2, and for the 1QP sector (the 2QP sector can be worked out in a similar way). The aim is to compute hopping amplitudes, as those given in Appendix A 1. As can be seen in Fig. 10, one difference compared to the $0 \mathrm{QP}$ sector is that depending on which term of the Hamiltonian one computes, the particle can hop to different final positions. In the figure, we have again not represented any arrow to make things light, but it should be clear that the reference state is a ferromagnetic state, with one spin flip at the QP's position. We have only considered hoppings $t_{i, j}$ of $i$ sites in $x$ direction and $j$ sites in $y$ direction, for which $i \geqslant j \geqslant 0$, other processes can be found thanks to symmetries of the square lattice and the hermiticity of the Hamiltonian. The hopping amplitude $t_{0,0}$, which should rather be called a chemical potential, is a bit peculiar. First, the counting operator $Q$ appearing in $H_{\text {eff }}$ gives a contribution of 4 . Second, as one is only interested in the excitation energy above the ground state, the $0 \mathrm{QP}$ contributions of the clusters have to be subtracted.

Various contributions of Fig. 10 can be extracted from the effective Hamiltonian's matrix elements which are shown in Figs. 11 and 12. Again, one can invert these relations, which leads to a recursive subcluster subtraction scheme (see Figs. 13 and 14). It is not as easy as in the 0QP sector to write down a concise formula similar to Eqs.(24) and (26), because the particle position has to be taken in consideration, and implies some more restrictions. However things should be clear from Figs. 11 and 12 . In practice, it is much easier to use a computer program that determines all relevant subclusters and subtracts their contributions.

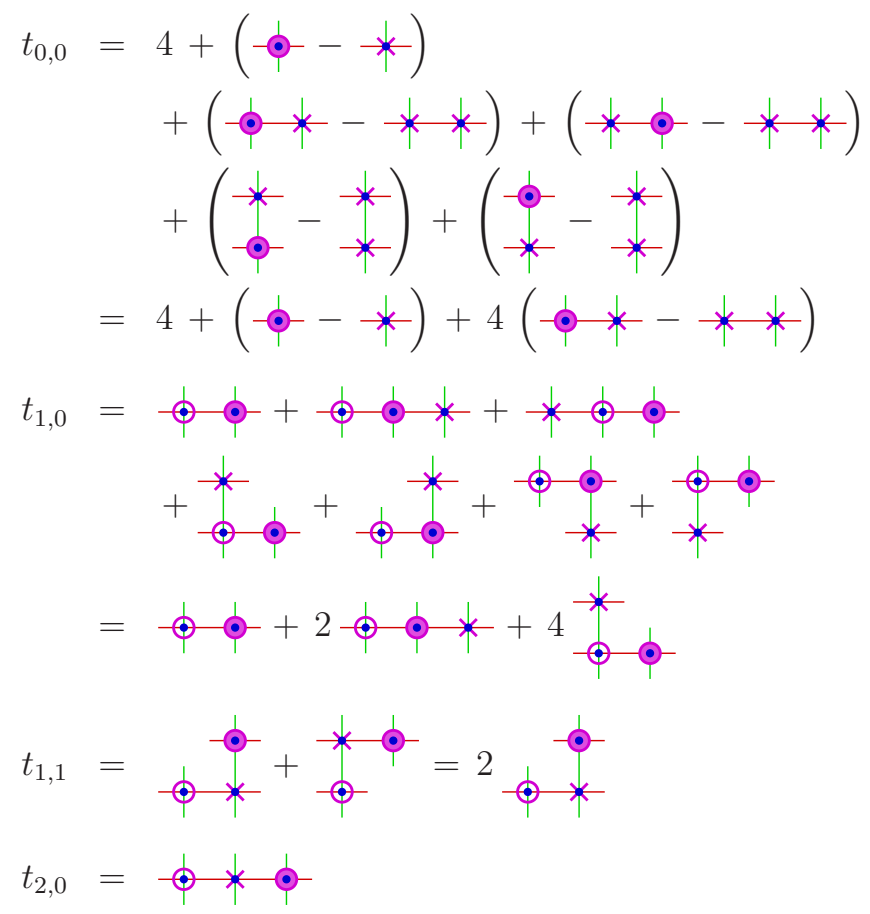

FIG. 10: (Color online) Contributions to hopping amplitude at order 2, as a linked-cluster expansion. Crosses have the same meaning as in Fig. 5, and are acted upon at least twice by $T_{n}$ operators. The empty (full) circle represents the initial (final) position of the particle. Sites with circles are acted upon at least once by $T_{n}$ operators. When the "particle" does not move, $i$. e., for $t_{0,0}$, the particle's site (only represented with a full circle) is acted upon at least twice by $T_{n}$ operators. In this case, as one is only interested in the energy of a 1QP state with respect to the $0 \mathrm{QP}$ ground state, one must subtract the 0QP amplitude (Refs. 18,19 and 23). Note that for $t_{0,0}$, one should also not forget the action of the counting operator $Q$, which gives a zeroth-order contribution equal to 4 .

As a final word, let us mention that the 2QP sector can be treated in the same way since the bound states in the TFIM or the Heisenberg model, that are of interest to us, behave as a single particle (they are made of two nearest-neighbor magnons). If one was interested in the 2QP sector where the two magnons can move freely, one would not only need to perform the 0QP subtraction as previously, but one would also have to subtract the two $1 \mathrm{QP}$ contributions, in order to extract the 2QP interactions. We shall now apply this formalism to both systems introduced in Sec. II.

\section{RESULTS FOR TFIM}

\section{A. Series expansion of the low-energy spectrum}

As we have seen, PCUTs provide, order by order, an effective Hamiltonian $\widetilde{H}_{\text {eff }}^{\text {TFIM }}$ which is unitarily equivalent to $\widetilde{H}_{\text {TFIM }}$ and commutes with the operator $Q$ counting 


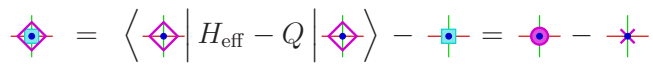

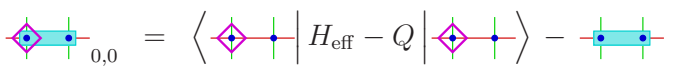

$$
\begin{aligned}
& =-\bullet *+-\bullet \cdot++*-(* *+2 *) \\
& =(-\bullet *-* *)+(-\phi-*)
\end{aligned}
$$

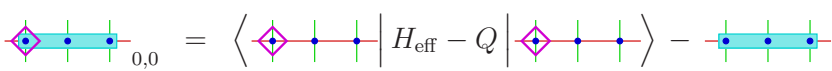

$$
\begin{aligned}
& =-\phi * \cdot++* * *+-\phi \cdot \cdot \\
& +* *+++\cdot *+(-\phi * *)^{(3)} \\
& -\left((* * *)^{(3)}+2 * *+3 *\right) \\
& =(-\bullet-*)+(-\bullet *-* *) \\
& +(-\bullet * *-* * *)^{(3)}
\end{aligned}
$$

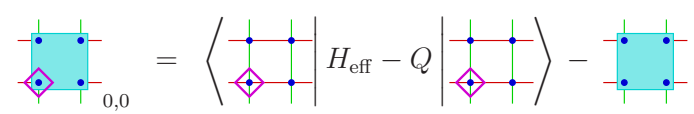

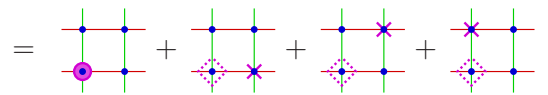

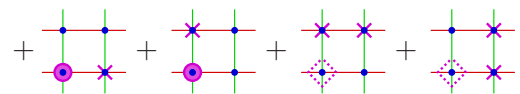

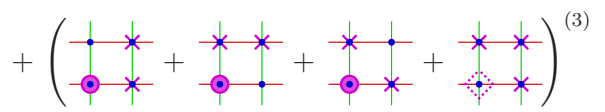

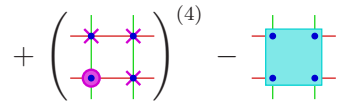

$$
\begin{aligned}
& =(-\phi-*)+2(-\phi *-* *) \\
& +(\ldots)^{(3)}+(\ldots)^{(4)}
\end{aligned}
$$

FIG. 11: (Color online) Matrix elements of $H_{\text {eff }}$ between identical initial and final states that are useful for the computation of $t_{0,0}$ at order 2. Diamonds with solid lines represent the particle position in the considered state. Contributions arising from an action of $H_{\text {eff }}-Q$ which does not involve the particle site contain a dotted diamond that keeps trace of this site. Other symbols have the same meaning as in previous figures. Terms in parenthesis are higher-order terms, for which the exponent gives the lowest order they start to contribute. For the last contribution, intermediate steps have not been shown, and the higher-order terms are not kept until the end, to keep things as light as possible.

the number of antiferromagnetic bonds. One must then analyze $\widetilde{H}_{\text {eff }}^{\text {TFIM }}$ in each sector with a given number $q$ of antiferromagnetic bonds. In the present work, we derived this effective Hamiltonian in the sector $q=0$ (0qp or $0 \mathrm{QP}$ ) up to order 14 while we reached order 12 for $q=4$ (4qp corresponding to $1 \mathrm{QP}$ ) and $q=6$ (6qp corresponding to a bound state of $2 \mathrm{QP}$ ). We restrict ourselves to the "physical" subspace (i.e., the states corresponding to magnons) where all conserved quantities $\left(B_{p}\right.$ 's) of Sec. IID are set to +1 , and postpone the discussion of

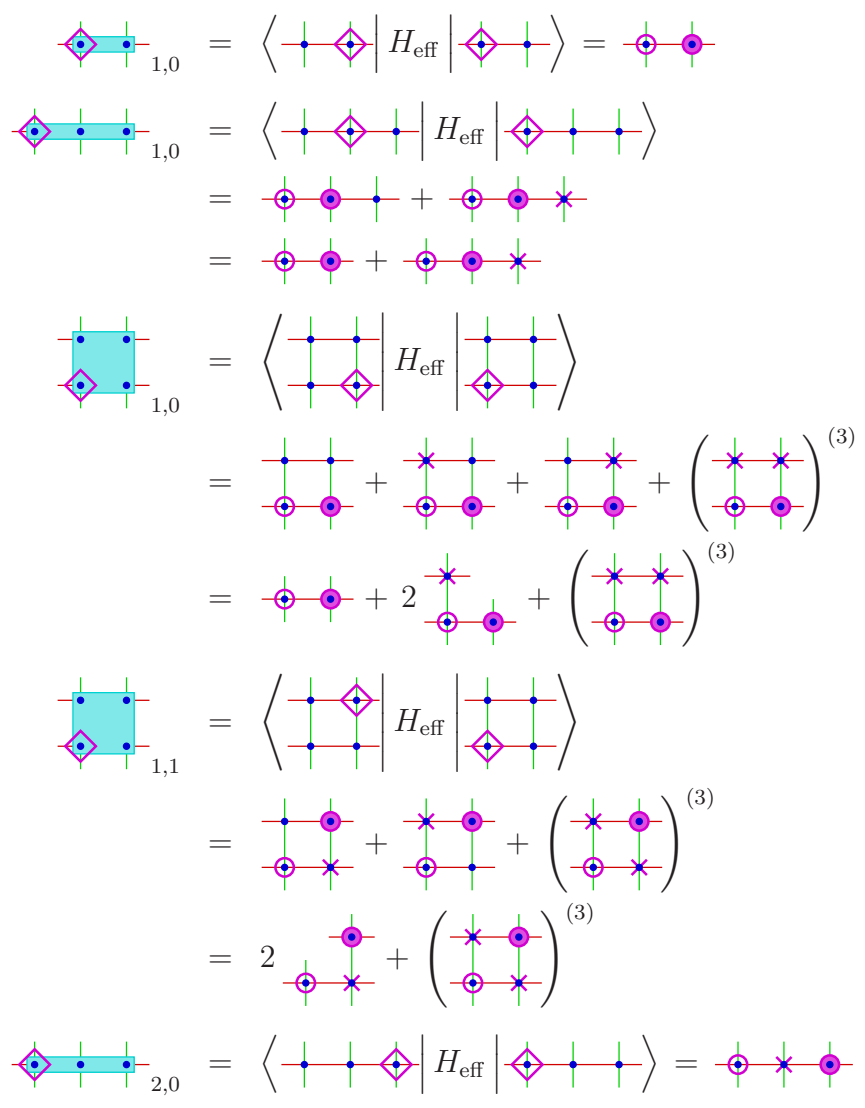

FIG. 12: (Color online) Matrix elements of $H_{\text {eff }}$ between different initial and final states that are useful for the computation of $t_{1,0}, t_{2,0}$, and $t_{1,1}$ at order 2 . Conventions are the same as in Fig. 11.

"unphysical" states (having antiferromagnetic bonds not corresponding to a magnon configuration of the initial models) to Sec. VII.

For $q=0$, we obtain the ground-state energy per bond

$$
\begin{aligned}
e_{0}= & -\frac{1}{2}-\frac{1}{8} h^{2}-\frac{1}{384} h^{4}-\frac{1}{6144} h^{6}-\frac{181}{3538944} h^{8} \\
& -\frac{1388129}{254803968000} h^{10}-\frac{67647506447}{25684239974400000} h^{12} \\
& -\frac{707258321166713}{2588971389419520000000} h^{14},
\end{aligned}
$$

which matches with the result given in Ref. ${ }^{28}$.

The first nontrivial "physical" sector for the TFIM is the one-magnon sector, which is a peculiar case of $q=4$, since the four antiferromagnetic bonds must have a relative position similar to the one shown in Fig. 2. We computed the dispersion $\varepsilon_{4}\left(k_{x}, k_{y}\right)$ up to order 12 . The list of all relevant hopping amplitudes is given in Appendix A 1 . Noting that $\varepsilon_{4}\left(k_{x}, k_{y}\right)$ is minimal for $\left(k_{x}, k_{y}\right)=(0,0)$, one 


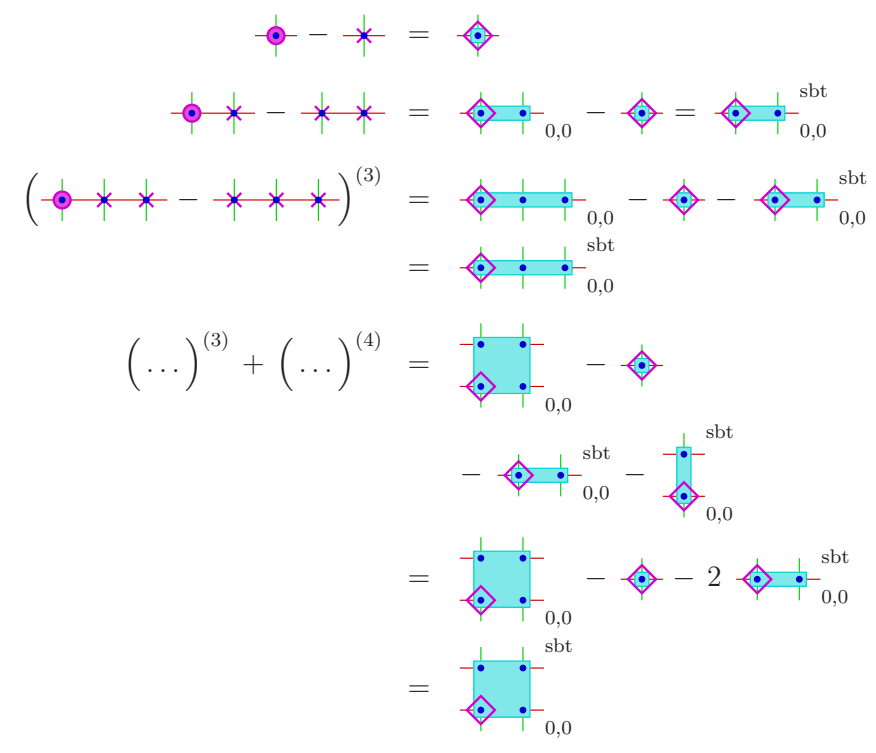

FIG. 13: (Color online) Inversion of relations shown in Fig. 11. The exponent sbt (for subtracted) refers to the subcluster subtraction.

gets the one-magnon gap

$$
\begin{aligned}
\Delta_{4}= & 4-\frac{3}{2} h^{2}+\frac{43}{96} h^{4}-\frac{19993}{27648} h^{6} \\
& +\frac{82873487}{79626240} h^{8}-\frac{1901437203257}{1146617856000} h^{10} \\
& +\frac{64764934458802909}{23115815976960000} h^{12} .
\end{aligned}
$$

Note that our results also match those given in Refs. 19 and 28 up to some trivial rescaling of the Hamiltonian's parameters.

The next "physical" sector for the TFIM is a subspace of the 6qp sector which corresponds to the bound states discussed above and is illustrated in Fig. 3. In this sector, the effective Hamiltonian describes the hopping of the bound state, whose center of mass lives on the square lattice formed by the middles of the bonds (dots in Fig. 4). However, one must state that there are two different types of sites in this lattice (see Fig. 4) since bonds can be vertical or horizontal. This is obvious in Fig. 3 where the two bound states involve a different pattern of antiferromagnetic bonds. Series expansion of the hopping amplitudes are given in Appendix A 2 up to order 12. From these amplitudes it is clear that the bound state does not have the same probability to hop in a given direction, for instance $x$, if it is on a horizontal or on a vertical bond. Of course, this important distinction also holds for the XXZ model discussed in the next section.

Therefore, one is led to diagonalize a $2 \times 2$ matrix for each value of the center of mass momentum $\boldsymbol{k}=\left(k_{x}, k_{y}\right)$. One can in particular extract the gap, which is found to

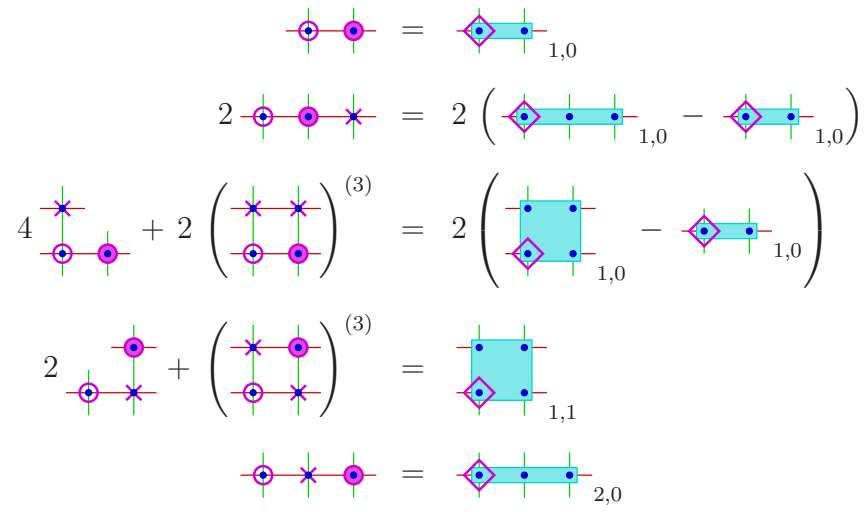

FIG. 14: (Color online) Inversion of relations shown in Fig. 12.

be the lowest of the two energies at $\boldsymbol{k}=(0,0)$, and reads

$$
\begin{aligned}
\Delta_{6}^{-}= & 6-\frac{275}{96} h^{4}-\frac{11521}{27648} h^{6}+\frac{16400551}{7962624} h^{8} \\
& +\frac{1459322986427}{143327232000} h^{10}-\frac{101780777359633847}{28894769971200000} h^{12}
\end{aligned}
$$

The energy of the second mode at zero center of mass momentum is higher and given by

$$
\begin{aligned}
\Delta_{6}^{+}= & 6-\frac{11}{96} h^{4}-\frac{115}{1024} h^{6}-\frac{4956689}{39813120} h^{8} \\
& -\frac{1720028423}{17915904000} h^{10}-\frac{880952915946869}{9631589990400000} h^{12} .
\end{aligned}
$$

We do not analyze the associated bound state in detail, but just note that this mode decays into the two-magnon continuum well before the critical point. Physical implications of such a decay will be discussed for the XXZ model below.

\section{B. Exact diagonalization}

To have some finite-size crosschecks of the validity of the perturbative expansions and the mapping to the Kitaev-type Hamiltonians, performing exactdiagonalization (ED) studies on the microscopic models defined above demands particular adjustment. First, the appearing interactions are of four-body type for TFIM in Eq. (10) and of seven-body type for XXZ in Eq. (11). Second, the amount of symmetries is enormous, which enables us to go to big systems as up to $N=50$ sites. This, however, demands a suitable way to generate the symmetrically reduced basis, as a loop through all possible spin configurations would be impracticable. To overcome the first problem, we apply the Kernel sweeping method to efficiently implement many-body interaction terms, where details are elaborated on in Ref. 45. Basically, a small $m$-site Hamiltonian of the $m$-body interaction is defined, and implemented to sweep over the whole lattice basis. To address the second point, we do 
not loop over all possible $N$-site configurations and subsequently sort out by symmetry constraints, but start with an allowed state of the basis and iteratively act on it by the respective Hamilton operator. In each step, new unprecedented scattering states are collected and added to the yet incomplete basis, until a new action of $H$ does not produce new scattering states anymore. In doing so, as $H$ commutes with the $\mathbb{Z}_{2}$ symmetries Eqs. (12) and (14), we make sure that the iteration procedure only acts within the $\mathbb{Z}_{2}$ charge subsector we want to consider. For $N=50$, this yields a subblock dimension of $2^{50 / 2-1}=16777216$ as alluded to previously, which is already in suitable range for Lanczos diagonalization algorithms. Still, from there, we can further exploit lattice symmetries, such as the translational invariance of the models, to specify the point of the Brillouin zone we want to study. For the Hamiltonians considered, we compute the low-energy spectra for clusters up to $N=50$ sites. As shown in Fig. 15, the finite-size corrections do not allow us to adequately describe the vicinity of the intermediate and large $h$ limit, as the proximity of the continuum and the bound-state modes becomes comparable to the finitesize splitting scale. Apparently, Fig. 15 explicates that the ED data for the largest available system size corresponds to the expansion data up to $h \sim 0.6$. However, in the regime where the perturbative expansion data likewise starts to fluctuate a lot depending on the order of the expansion, the ED data cannot be used for suitable analysis. In the following, it is thus implicitly assumed that we use exact diagonalization to crosscheck the implementation of the perturbative expansions, but can only rely on the latter to highest order to study the interesting regimes where the bound-state modes approach the continuum.

\section{Analysis and gap ratio at the critical point}

As already discussed in Ref. 28, the series expansion for $\Delta_{4}$ is strongly diverging so that one has to perform some resummation to extract gap values for finite $h$. This also seems to be the case for $\Delta_{6}^{-}$, although we only have coefficients up to order 12 for this quantity. Unfortunately, standard Padé-type resummation procedure using the order 20 expansion for $\Delta_{4}$ given in Ref. 28 leads to a critical point which is still far from the most accurate value. Indeed, naive Padé approximants ([10,10], $[8,12],[12,8])$ lead to $h_{c}=(1.65045,1.57029,1.73306)$, respectively, whereas the position of the critical point computed from series expansion in the high-field phase ${ }^{27}$ is $h_{c}=1.5216(6)$. Note that for its classical counterpart which is the critical temperature, higher precision of order $10^{-7}$ has been reached (see, for instance, Ref. 46 and references therein).

Here, our aim is to check a result coming from field-theoretical calculations performed by Caselle et al. ${ }^{16,17}$ who predict that $\Delta_{6}^{-} /\left.\Delta_{4}\right|_{h=h_{c}} \simeq 1.8$. Recently, this prediction has been improved using numerical

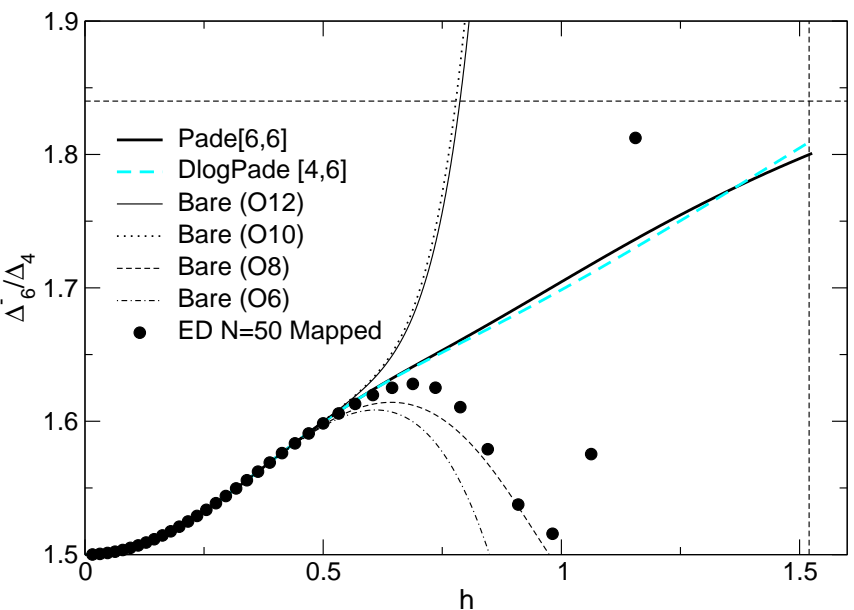

FIG. 15: (Color online) Thin lines represent the bare ratio $\Delta_{6}^{-} / \Delta_{4}$ for different maximal orders $6,8,10$, and 12 as a function of the magnetic field $h$. Thick lines correspond to different approximations of this ratio. Filled circles denote ED data of the mapped model (10) with $N=50$ sites (bonds of the original lattice). Dashed vertical (horizontal) line marks the value of the magnetic field (ratio) at the critical point as obtained from the numerical diagonalizations of Ref. 47.

diagonalization ${ }^{47}$, giving a ratio of $1.84(3)$.

Given that the transition point is defined by $\Delta_{4}=0$, a finite ratio at the critical field means that (i) $\Delta_{6}^{-}=0$ at this point, and (ii) $\Delta_{6}^{-}$vanishes with the same critical exponent $\nu$. It can therefore be expected that the direct extrapolation of $\Delta_{6}^{-}$is at least as complicated as the one for $\Delta_{4}$ which is actually the case. By contrast, one may hope that the ratio $\Delta_{6}^{-} / \Delta_{4}$, on which we focus below, has a better behavior.

The bare series of the ratio $\Delta_{6}^{-} / \Delta_{4}$ up to order 12 reads

$$
\begin{aligned}
\frac{\Delta_{6}^{-}}{\Delta_{4}}= & \frac{3}{2}+\frac{9}{16} h^{2}-\frac{517}{768} h^{4}-\frac{32831}{221184} h^{6} \\
& +\frac{156729359}{637009920} h^{8}+\frac{27593405457803}{9172942848000} h^{10} \\
& -\frac{415396528829457211}{924632639078400000} h^{12},
\end{aligned}
$$

and is shown in Fig. 15. Clearly, the bare series is still alternating and the convergence is rather poor $(h \leq 0.5)$ so extrapolation schemes are mandatory. First we tried standard Padé approximants. We found that all approximants $[n, m]$ with $n+m \leq 10$ give no useful result in the sense that the approximant either has a spurious pole or shows a diverging behavior well before the critical field $h_{c}$. Looking at the Padé approximants with $n+m=12$ we found only two valid cases, namely, $[8,4]$ and $[6,6]$. Still no converging picture emerges because the $[8,4]$ approximant displays a diverging behavior. However, the approximant $[6,6]$ is the first one to behave smoothly (see Fig. 15). We conclude that the Padé analysis gives no convergent picture and it seems that one needs at least 
the order 12 series to catch the physics of this ratio close to the critical point.

To proceed further, we used DlogPadé extrapolation which is usually more reliable than Padé extrapolations for positive quantities. Among all approximants with $n \geq$ 4 and $m \geq 4$, DlogPadé $[4,6]$ is the only one that has no spurious pole. This extrapolation is shown in Fig. 15. It can be seen that the ratio seems to behave almost linearly as a function of the field close to the critical point. The ratio at the critical field $h_{c}$ is 1.81 which is very close to the numerical value ${ }^{47}$. So one finds again that one needs at least order 12 to capture the expected behavior. Clearly, higher orders are expected to give more valuable insights in this quantity but these are beyond the scope of this work.

The relevance of higher orders can be also understood from the fact that fluctuations on rather large length scales are required to follow this ratio up to the critical point. This is in agreement with ED results, displayed in Fig. 15, which are qualitatively similar to the bare PCUTs series.

\section{RESULTS FOR THE XXZ MODEL}

\section{A. Series expansion of the low-energy spectrum}

Similarly, we computed the low-energy spectrum of the XXZ model by the PCUTs method. As previously, we restrict the discussion again to the "physical" subspaces $q \in\{0,4,6\}$ corresponding to the ground state, to one-magnon states and to two-magnon bound states. We derive the effective Hamiltonian up to order 10 in the $0 \mathrm{QP}$ sector and up to order 8 in the $1 \mathrm{QP}$ and $2 \mathrm{QP}$ sectors.

For $q=0$, we obtain the ground-state energy per dimer

$$
\begin{aligned}
e_{0}= & -\frac{1}{2}-\frac{1}{6} \lambda^{2}+\frac{1}{1080} \lambda^{4}-\frac{3587}{2268000} \lambda^{6} \\
& -\frac{660294389}{800150400000} \lambda^{8}-\frac{156875294970593831}{503046234915840000000} \lambda^{10},
\end{aligned}
$$

which matches with the result given in Ref. 29.

The one-magnon sector corresponds again to the first nontrivial "physical" sector with $q=4$. The four antiferromagnetic bonds must remain in a closed-pack relative position such that they share a site in the original square lattice (see Fig. 2). The dispersion can be obtained from the list of hopping amplitudes given in Appendix B 1 . The minimum of the dispersion is found at $\boldsymbol{k}=(0,0)$, and one gets for the one-magnon gap, in accordance with Ref. 30,

$$
\begin{aligned}
\Delta_{4}= & 4-\frac{10}{3} \lambda^{2}+\frac{137}{216} \lambda^{4}-\frac{13039847}{15552000} \lambda^{6} \\
& +\frac{124898889761701}{230443315200000} \lambda^{8} .
\end{aligned}
$$

We now switch to the $q=6$ sector that corresponds to the two-magnon bound state. Its analysis follows exactly the same steps as for the TFIM discussed in the previous section. Series expansions of the hopping amplitudes for the bound state are given in Appendix B 2 up to order 8. One has to diagonalize a $2 \times 2$ matrix for each value of the center-of-mass momentum $\boldsymbol{k}=\left(k_{x}, k_{y}\right)$. The gap is found at $\boldsymbol{k}=(0,0)$, and reads

$$
\begin{aligned}
\Delta_{6}^{-}= & 6-\frac{10}{3} \lambda^{2}+\frac{323}{540} \lambda^{4}-\frac{1435321}{324000} \lambda^{6} \\
& +\frac{3809941658983}{320060160000} \lambda^{8} .
\end{aligned}
$$

The other bound mode at higher energy with the same momentum $\boldsymbol{k}=(0,0)$ has the expansion

$$
\begin{aligned}
\Delta_{6}^{+}= & 6+\frac{2}{3} \lambda^{2}-\frac{619}{1080} \lambda^{4}-\frac{482989}{1036800} \lambda^{6} \\
& -\frac{15320370383651}{19203609600000} \lambda^{8} .
\end{aligned}
$$

We would like to point out that the two different orientations of the bound state are missed in Ref. 15. As a consequence, the dispersion for arbitrary momentum of the bound state is not correct in this reference. Only the gap in this sector matches with our results (which certainly means that all hopping amplitudes in Ref. 15 are correct).

\section{B. Fate of the bound states}

An interesting question is to determine when the twomagnon bound states decay as a function of $\lambda$. To this end, we first focus on the case of total momentum $\boldsymbol{k}=(0,0)$, describing low-energy physics. Bound states decay for this momentum at latest at the Heisenberg point $\lambda=1$, where the one-magnon gap closes and therefore all multimagnon continua have a gapless spectrum.

The lowest energy of the two-magnon continuum is found at total momentum $\boldsymbol{k}=(0,0)$ and is given by twice the one-magnon gap $\Delta_{4}$. Bound states start to acquire a finite lifetime once their energy is degenerate with the lower band edge of the two-magnon continuum. Consequently, we determine the values of $\lambda$ for which ratios $2 \Delta_{4} / \Delta_{6}^{ \pm}$are equal to one. In the following, we restrict the discussion to the PCUTs results since reliable ED results would require enormous system sizes (with 36 sites one would only capture terms of order $\lambda^{2}$ ). The bound state is indeed an extended object and the $\lambda$ term in the XXZ Hamiltonian (6) involves nearest-neighbor interactions, contrary to the purely local $h$ term in the TFIM Hamiltonian (1).

Obviously, the high-energy mode $\Delta_{6}^{+}$decays first. Using different extrapolation schemes such as Padé and DlogPadé, we find that it disappears for $\lambda \simeq 0.5401(1)$, $i$. e., for a rather small value which explains the high accuracy. Beyond this point, the bound state gains a finite lifetime and, strictly speaking, a perturbative derivation 


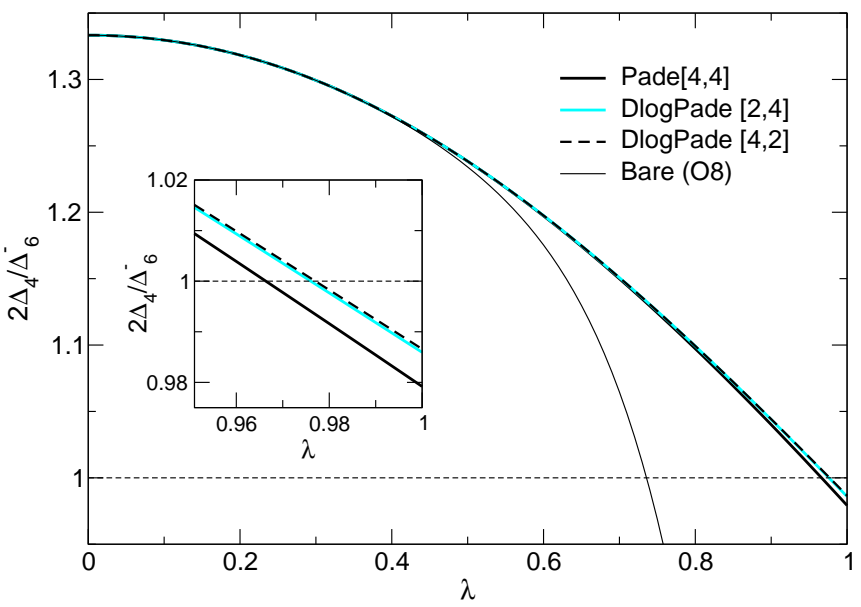

FIG. 16: (Color online) Thin blue line represents the bare ratio $2 \Delta_{4} / \Delta_{6}^{-}$for the maximal order 8 as a function of the anisotropy parameter $\lambda$. Thick lines correspond to different approximations of this ratio. The dashed horizontal line marks the value 1 of the ratio. The value $\lambda=1$ corresponds to the Heisenberg point.

of a block-diagonal Hamiltonian becomes impossible ${ }^{48}$. One can expect that the decayed bound state shows up as resonances inside the continua of dynamical correlation functions. The two-magnon peak observed in the theoretical Raman response at the Heisenberg point $\lambda=1$ (Refs. 49 and 50) and experimentally detected in the undoped cuprate compounds ${ }^{51,52}$ might be a remnant of this bound state. However, a correct physical description of the decay process is beyond any series expansion study. This scenario is further confirmed by the fact that any Padé extrapolation of the energy $\Delta_{6}^{+}$has poles in the denominator.

Concerning the low-energy properties, in analogy to the Ising case studied in the previous section, the fate of the low-energy mode $\Delta_{6}^{-}$is more interesting but also more challenging. The bare ratio $2 \Delta_{4} / \Delta_{6}^{-}$together with different approximants are shown in Fig. 16. We observe that no pole shows up in these approximants. Consistently, all approximations indicate that the decay takes place very close to the Heisenberg point $(\lambda \simeq 0.97)$. Taking into account that the series have been obtained up to order 8 only and have only even orders, one cannot tell precisely whether the merging point is located exactly at the critical point $(\lambda=1)$, just as in the TFIM, or not. One argument in favor of the former scenario is that, for any finite-size system, the ground state of the SU(2)-invariant Heisenberg point is a singlet and the gapped elementary excitation is a threefold degenerate triplet, with total magnetization $S_{z} \in\{-1,0,+1\}$. The excitations with $S_{z} \in\{-1,+1\}$ can be identified with one-magnon excitations found for $\lambda<1$, whereas the excitation with $S_{z}=0$ has to be a two-magnon bound state. The gap of these excitations should furthermore vanish in the thermodynamical limit considered in the present PCUTs study.

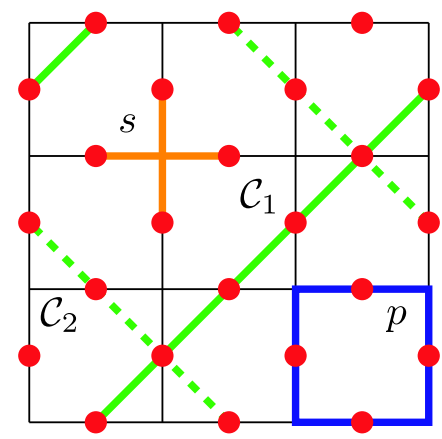

FIG. 17: (Color online) Square lattice on which the toric code Hamiltonian (36) is defined with periodic boundary conditions. Sites are represented with dots. We also show a vertex $s$, a plaquette $p$, a diagonal contour $\mathcal{C}_{1}$, and an antidiagonal contour $\mathcal{C}_{2}$, used to define various operators (see text).

\section{THE TORIC CODE MODEL IN A TRANSVERSE FIELD WITH $\mathrm{J}_{\mathrm{p}}=\mathbf{0}$}

As mentioned in Sec. IID, the toric code Hamiltonian (10) deserves to be analyzed on its own. Indeed, in the original toric code model ${ }^{25}$, Kitaev focused on the isotropic coupling $J_{\mathrm{s}}=J_{\mathrm{p}}$ [see Eq. (13) for notations]. Here, the bond description of the TFIM leads us to consider a different situation where $(i) J_{\mathrm{p}}=0$ and $(i i)$ a magnetic field in the $z$ direction is introduced. Let us stress that such a model is very close to the Xu-Moore model $^{53}$ and, in some sense, very similar to the parallelfield problem discussed in Refs. 32 and 26,33,34. Most importantly, it is exactly the model introduced by Wegner in his seminal paper ${ }^{54}$ (see also Ref. 33) but, here, $B_{p}$ operators are conserved quantities that can be in any configuration. This crucial difference raises several questions that we shall address in the present section.

Let us first rewrite the Hamiltonian and various operators of Sec. II D in the toric code language. The Hamiltonian reads

$$
H=-J \sum_{s} A_{s}-h \sum_{i} \sigma_{i}^{z}
$$

where the spins live on the bonds of a square lattice (see Fig. 17) and the $A_{s}=\prod_{i \in s} \sigma_{i}^{x}$ operators involve the four sites around a vertex $s$. The plaquette operators $B_{p}=\prod_{i \in p} \sigma_{i}^{z}$ are conserved. For a system defined with periodic boundary conditions the cycle operators $\prod_{i \in \mathcal{C}} \sigma_{i}^{z}$ defined on diagonal or antidiagonal contours, such as those shown in Fig. 17, are conserved as well.

The main difference with the $\mathrm{Xu}$-Moore Hamiltonian ${ }^{53}$ is that $A_{s}$ operators only act on vertices of the square lattice and not on plaquettes. Furthermore, in the $\mathrm{Xu}$ Moore model, the cycle operators are still conserved, contrary to the $B_{p}$ operators. The dynamics of the quasiparticles is thus expected to be more constrained than in the $\mathrm{Xu}$-Moore model which already exhibits dimensional reduction. 
In the following, we shall discuss separately the two limits (large and small $J / h$ ) for which we computed perturbatively the low-energy spectrum. One can already note that in the small-field limit, the system is in a topological phase (i. e., the ground-state degeneracy depends on the surface genus) whereas at large field the ground state is obviously unique. Thus, one expects a (topological) quantum phase transition when varying the ratio $J / h$.

\section{A. Large-field limit $h \gg J$}

For $J=0$, elementary excitations are usual magnons obtained by flipping any spins from the ground state which is the state fully polarized in the field $(z)$ direction. PCUTs formalism will then give a dressed magnon description when switching on $J^{26}$. Setting $h=1 / 2$, the ground-state energy per bond (which coincides with the 0qp level) is obtained from Eq. (27) by replacing $h$ by $J$.

However, for this model, arbitrary qp sectors are allowed although conservation of $B_{p}$ 's imposes severe constraints on the spectrum. For $q=1$, one only has a nondispersive (excited) level at energy

$$
\begin{aligned}
\Delta_{1}= & 1-\frac{J^{2}}{2}+\frac{3 J^{4}}{32}-\frac{31 J^{6}}{768}+\frac{299233 J^{8}}{15925248} \\
& -\frac{2014178639 J^{10}}{764411904000},
\end{aligned}
$$

since any displacement of this localized dressed magnon would modify the $B_{p}$ 's configuration.

For $q=2$, the only possible dynamics is provided by some flip-flop processes depicted in Fig. 18 (we do not consider the case where the two dressed magnons are far apart). Both configurations shown in Fig. 18 yield the same gap, which reads

$$
\begin{aligned}
\Delta_{2}= & 2-J-\frac{J^{2}}{4}+\frac{J^{3}}{8}-\frac{13 J^{4}}{192}+\frac{29 J^{5}}{768}-\frac{445 J^{6}}{13824} \\
& +\frac{739 J^{7}}{36864}-\frac{608839 J^{8}}{79626240}-\frac{2462069 J^{9}}{9555148800} \\
& +\frac{21097903 J^{10}}{152882380800} .
\end{aligned}
$$

Note that in the Xu-Moore model, the pair of nearestneighbor magnons (top of Fig. 18) is allowed to hop at any distance (but in a one-dimensional stripe) ${ }^{14}$.

We have not computed energies of states with more magnons. Let us simply mention the following two points. For $q=3$, the problem is similar to $q=2$ and no dynamics of the QPs is allowed apart from flip-flip processes. The first dispersive mode is the one involving four magnons, just as in the $\mathrm{Xu}-\mathrm{Moore}$ model $^{14}$.

\section{B. Small-field limit $h \ll J$}

Let us now turn to the opposite limit where $J$ is much larger than $h$ and for which the system is in a topological
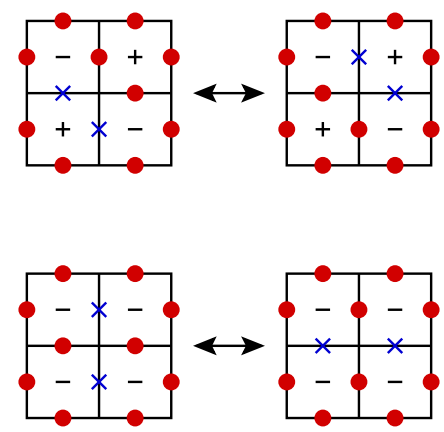

FIG. 18: (Color online) Illustration of the two-magnon dynamics in the high-field limit of Hamiltonian (36). Dressed magnons are depicted as crosses. The + and - signs refer to the eigenvalue +1 or -1 of the plaquette operators $B_{p}$.

phase. For convenience, let us set $J=1 / 2$. Since the $B_{p}$ operators do not appear in the Hamiltonian, all states are macroscopically degenerate when $h=0$. It is thus natural to wonder what the effect of the perturbation is. A calculation at fourth order of the effective Hamiltonian in the sector where there is no effective vertex excitation (still using the PCUTs formalism) yields

$$
H_{\mathrm{eff}}=-N\left(\frac{1}{4}+\frac{h_{z}^{2}}{2}+\frac{5 h_{z}^{4}}{8}\right)-\frac{5 h_{z}^{4}}{2} \sum_{p} B_{p}
$$

where $N$ is the number of sites (and thus twice the number of plaquettes or of vertices). We thus see that, once the magnetic field is switched on, the ground state belongs to the sector where each operator $B_{p}$ has eigenvalue +1 . As a check, note that setting all $B_{p}$ operators to one in the above formula gives the ground-state energy, which matches with Eq. (8) of Ref. 26 (apart from a trivial constant shift since no $B_{p}$ operator appears in our Hamiltonian, and after setting $h_{x}$ to zero in this equation). The fact that the sector with no plaquette excitation is selected by the perturbation is consistent with an Ising-type quantum phase transition. Indeed, in this sector, the model with Hamiltonian (36) is dual to the two-dimensional transverse-field Ising model (which follows from the same arguments as those used in Ref. 32).

\section{CONCLUSIONS}

We have studied two-magnon bound states in the TFIM and XXZ models using PCUTs around the Ising limit. This method gives a nice physical picture of these excitations in terms of dressed magnons which can be of two kinds on a square lattice depending on the "frustrated bond" localizing it (horizontal or vertical). Consequently, one obtains two different bound modes contrary to previous claims ${ }^{15,55}$. For both systems, we find that the lowest-energy gap vanishes at the critical point, or at least in a neighborhood that cannot be distinguished 
from this point with actual series expansions. Solving this issue, especially for the XXZ model, would clearly require much higher orders or a nonperturbative treatment as the one considered in Refs. 16 and 17 for the TFIM.

From a methodological point of view, we have adapted Entings' finite-lattice method commonly used in statistical mechanics to quantum problems. This approach basically consists in a cluster embedding which considerably increases the efficiency (from the time and memory point of view) of the PCUTs method we used here. Note that for the XXZ model, such an improvement allowed us to reach the same maximum order as standard series expansions techniques (see Refs. 19 and 15) based on a more sophisticated graph analysis. Clearly, adapting such a graph description to PCUTs is a crucial issue which is currently under study and should allow one to reach higher orders.

\section{Acknowledgments}

We wish to thank M. Caselle, M. D. Schulz, and G. S. Uhrig for fruitful discussions. K.P.S. acknowledges ESF and EuroHorcs for funding through his EURYI.

\section{Appendix A: Low-energy spectrum of the TFIM}

\section{One-magnon hopping amplitudes}

One-magnon excitations are located on sites of the square lattice. The corresponding hopping amplitudes $t_{i, j}$ of $i$ sites in $x$ direction and $j$ sites in $y$ direction are given below. Hopping amplitudes that are not given can be deduced from the symmetries of the lattice and the hermiticity of the Hamiltonian.

$$
\begin{aligned}
t_{0,0}= & 4-\frac{1}{2} h^{2}+\frac{19}{96} h^{4}-\frac{4745}{27648} h^{6}+\frac{15167827}{79626240} h^{8} \\
& -\frac{274582941007}{1146617856000} h^{10}+\frac{39052830905417587}{115579079884800000} h^{12} \\
t_{1,0}= & -\frac{1}{4} h^{2}+\frac{1}{16} h^{4}-\frac{785}{9216} h^{6}+\frac{49355}{442368} h^{8} \\
& -\frac{1325086777}{8493465600} h^{10}+\frac{503970370332103}{2140353331200000} h^{12} \\
t_{1,1}= & -\frac{29}{768} h^{6}+\frac{82181}{1327104} h^{8}-\frac{94291093}{955514880} h^{10} \\
& +\frac{620486307800173}{3852635996160000} h^{12} \\
t_{2,0}= & -\frac{7}{768} h^{6}+\frac{25789}{1327104} h^{8}-\frac{15624304847}{382205952000} h^{10} \\
& +\frac{1492786454328649}{19263179980800000} h^{12}
\end{aligned}
$$

$$
\begin{aligned}
& t_{2,1}=-\frac{23}{9216} h^{6}+\frac{11441}{1327104} h^{8}-\frac{17711235911}{764411904000} h^{10} \\
& +\frac{273663316786417}{5503765708800000} h^{12} \\
& t_{3,0}=-\frac{23}{27648} h^{6}+\frac{1529}{995328} h^{8}-\frac{5258321581}{1146617856000} h^{10} \\
& +\frac{202725363389893}{16511297126400000} h^{12} \\
& t_{2,2}=\frac{307}{884736} h^{8}-\frac{442674073}{127401984000} h^{10} \\
& +\frac{31180724115977}{2568423997440000} h^{12} \\
& t_{3,1}=\frac{307}{1327104} h^{8}-\frac{258078503}{127401984000} h^{10} \\
& +\frac{268024184026579}{38526359961600000} h^{12} \\
& t_{4,0}=\frac{307}{5308416} h^{8}-\frac{24494311}{84934656000} h^{10} \\
& +\frac{11181306857333}{11007531417600000} h^{12} \\
& t_{3,2}=-\frac{1621657}{12740198400} h^{10}+\frac{359038393}{339738624000} h^{12} \\
& t_{4,1}=-\frac{1621657}{25480396800} h^{10}+\frac{54196069259}{122305904640000} h^{12} \\
& t_{5,0}=-\frac{1621657}{127401984000} h^{10}+\frac{11111462099}{203843174400000} h^{12} \\
& t_{3,3}=\frac{63560779}{1834588569600} h^{12} \\
& t_{4,2}=\frac{63560779}{2446118092800} h^{12} \\
& t_{5,1}=\frac{63560779}{6115295232000} h^{12} \\
& t_{6,0}=\frac{63560779}{36691771392000} h^{12}
\end{aligned}
$$

\section{Two-magnon bound state hopping amplitudes}

The two-magnon bound states are located at the centers of the bonds on the square lattice and exist in two kinds : the bound state can indeed live on a horizontal or a vertical link denoted $\mathrm{h}$ and $\mathrm{v}$ respectively. The hopping amplitudes of a horizontally oriented bound state $t_{i, j}^{\mathrm{h}, \alpha}$ are listed below. The corresponding hopping elements for the vertically oriented bound state can be deduced by reversing the $x$ and $y$ components, $i . e ., t_{i, j}^{\mathrm{v}, \alpha}=t_{j, i}^{\mathrm{h}, \alpha}$. Again, the symmetries of the lattice and the hermiticity of the Hamiltonian have been used to restrict the number of given amplitudes.

$$
\begin{aligned}
t_{0,0}^{\mathrm{h}, \mathrm{h}}= & 6-\frac{5}{8} h^{4}-\frac{8573}{27648} h^{6}+\frac{28100449}{79626240} h^{8} \\
& +\frac{34908351643}{23887872000} h^{10}+\frac{8582632522763479}{23115815976960000} h^{12}
\end{aligned}
$$




$$
\begin{aligned}
& t_{0,1}^{\mathrm{h}, \mathrm{h}}=-\frac{1}{3} h^{4}-\frac{1}{12} h^{6}+\frac{8209}{41472} h^{8}+\frac{4323615563}{4777574400} h^{10} \\
& +\frac{844706924968673}{8255648563200000} h^{12} \\
& t_{\frac{1}{2}, \frac{1}{2}}^{\mathrm{h}, \mathrm{v}}=-\frac{1}{3} h^{4}-\frac{59}{768} h^{6}+\frac{1052251}{4976640} h^{8}+\frac{266099856239}{286654464000} h^{10} \\
& +\frac{7513297407630751}{115579079884800000} h^{12} \\
& t_{1,0}^{\mathrm{h}, \mathrm{h}}=-\frac{1}{12} h^{4}+\frac{7}{192} h^{6}+\frac{102731}{2211840} h^{8}+\frac{5273001593}{15925248000} h^{10} \\
& -\frac{818755629011689}{3210529996800000} h^{12} \\
& t_{0,2}^{\mathrm{h}, \mathrm{h}}=\frac{1}{864} h^{6}+\frac{207833}{3981312} h^{8}+\frac{41166508631}{286654464000} h^{10} \\
& -\frac{687140651194889}{8255648563200000} h^{12} \\
& t_{\frac{1}{2}, \frac{3}{2}}^{\mathrm{h}, \mathrm{v}}=-\frac{1}{192} h^{4}+\frac{7}{384} h^{6}+\frac{94081}{2654208} h^{8}+\frac{118209917}{707788800} h^{10} \\
& -\frac{993567226390117}{6421059993600000} h^{12} \\
& t_{1,1}^{\mathrm{h}, \mathrm{h}}=-\frac{1}{192} h^{4}+\frac{43}{1536} h^{6}+\frac{5731}{294912} h^{8}+\frac{556424309}{3185049600} h^{10} \\
& -\frac{189675771174199}{917294284800000} h^{12} \\
& t_{\frac{3}{2}, \frac{1}{2}}^{\mathrm{h}, \mathrm{v}}=-\frac{1}{192} h^{4}+\frac{7}{384} h^{6}+\frac{94081}{2654208} h^{8}+\frac{118209917}{707788800} h^{10} \\
& -\frac{993567226390117}{6421059993600000} h^{12} \\
& t_{2,0}^{\mathrm{h}, \mathrm{h}}=-\frac{1}{192} h^{4}+\frac{13}{1536} h^{6}-\frac{199}{18432} h^{8}+\frac{20876171}{1061683200} h^{10} \\
& -\frac{2349250291300307}{23115815976960000} h^{12} \\
& t_{0,3}^{\mathrm{h}, \mathrm{h}}=-\frac{271}{1769472} h^{8}+\frac{23677}{19660800} h^{10} \\
& -\frac{471248557368467}{16511297126400000} h^{12} \\
& t_{\frac{1}{2}, \frac{5}{2}}^{\mathrm{h}, \mathrm{v}}=\frac{1}{1728} h^{6}-\frac{22393}{10616832} h^{8}+\frac{12078781789}{2293235712000} h^{10} \\
& -\frac{235040908701859}{5503765708800000} h^{12} \\
& t_{1,2}^{\mathrm{h}, \mathrm{h}}=\frac{1}{1728} h^{6}-\frac{15085}{5308416} h^{8}+\frac{1160612737}{229323571200} h^{10} \\
& -\frac{625401171948659}{8255648563200000} h^{12} \\
& t_{\frac{3}{2}, \frac{3}{2}}^{\mathrm{h}, \mathrm{v}}=\frac{1}{864} h^{6}-\frac{5939}{1327104} h^{8}+\frac{1534754177}{191102976000} h^{10} \\
& -\frac{470006557776271}{5778953994240000} h^{12} \\
& t_{2,1}^{\mathrm{h}, \mathrm{h}}=\frac{1}{864} h^{6}-\frac{5939}{1327104} h^{8}+\frac{77266223}{7644119040} h^{10} \\
& -\frac{23741703266443}{412782428160000} h^{12} \\
& t_{\frac{5}{2}, \frac{1}{2}}^{\mathrm{h}, \mathrm{v}}=\frac{1}{1728} h^{6}-\frac{22393}{10616832} h^{8}+\frac{12078781789}{2293235712000} h^{10} \\
& -\frac{235040908701859}{5503765708800000} h^{12}
\end{aligned}
$$

$t_{3,0}^{\mathrm{h}, \mathrm{h}}=\frac{1}{1728} h^{6}-\frac{203}{147456} h^{8}+\frac{872395631}{286654464000} h^{10}$ $-\frac{95952207356309}{11557907988480000} h^{12}$$$
t_{0,4}^{\mathrm{h}, \mathrm{h}}=\frac{7579}{331776000} h^{10}-\frac{1916092087}{7644119040000} h^{12}
$$$$
t_{\frac{1}{2}, \frac{7}{2}}^{\mathrm{h}, \mathrm{v}}=-\frac{271}{3538944} h^{8}+\frac{4822213}{10616832000} h^{10}
$$$$
-\frac{143095110961}{73383542784000} h^{12}
$$$$
t_{1,3}^{\mathrm{h}, \mathrm{h}}=-\frac{271}{3538944} h^{8}+\frac{3438923}{5308416000} h^{10}
$$$$
-\frac{3441481980911}{917294284800000} h^{12}
$$$$
t_{\frac{3}{2}, \frac{5}{2}}^{\mathrm{h}, \mathrm{v}}=-\frac{271}{1179648} h^{8}+\frac{29122303}{21233664000} h^{10}
$$$$
-\frac{7447108569091}{1223059046400000} h^{12}
$$$$
t_{2,2}^{\mathrm{h}, \mathrm{h}}=-\frac{271}{1179648} h^{8}+\frac{32991041}{21233664000} h^{10}
$$$$
-\frac{12599471323}{1791590400000} h^{12}
$$$$
t_{\frac{5}{2}, \frac{3}{2}}^{\mathrm{h}, \mathrm{v}}=-\frac{271}{1179648} h^{8}+\frac{29122303}{21233664000} h^{10}
$$$$
-\frac{7447108569091}{1223059046400000} h^{12}
$$$$
t_{3,1}^{\mathrm{h}, \mathrm{h}}=-\frac{271}{1179648} h^{8}+\frac{1683571}{1415577600} h^{10}
$$$$
-\frac{605405462489}{135895449600000} h^{12}
$$$$
t_{\frac{7}{2}, \frac{1}{2}}^{\mathrm{h}, \mathrm{v}}=-\frac{271}{3538944} h^{8}+\frac{4822213}{10616832000} h^{10}
$$$$
-\frac{143095110961}{73383542784000} h^{12}
$$$$
t_{4,0}^{\mathrm{h}, \mathrm{h}}=-\frac{271}{3538944} h^{8}+\frac{138329}{530841600} h^{10}
$$$$
-\frac{2796720985871}{3669177139200000} h^{12}
$$$$
t_{0,5}^{\mathrm{h}, \mathrm{h}}=-\frac{168493133}{45864714240000} h^{12}
$$$$
t_{\frac{1}{2}, \frac{9}{2}}^{\mathrm{h}, \mathrm{v}}=\frac{7579}{663552000} h^{10}-\frac{85039213657}{917294284800000} h^{12}
$$$$
t_{1,4}^{\mathrm{h}, \mathrm{h}}=\frac{7579}{663552000} h^{10}-\frac{12339018187}{91729428480000} h^{12}
$$$$
t_{\frac{3}{2}, \frac{7}{2}}^{\mathrm{h}, \mathrm{v}}=\frac{7579}{165888000} h^{10}-\frac{1225804705393}{3210529996800000} h^{12}
$$$$
t_{2,3}^{\mathrm{h}, \mathrm{h}}=\frac{7579}{165888000} h^{10}-\frac{1476569703919}{3210529996800000} h^{12}
$$$$
t_{\frac{5}{2}, \frac{5}{2}}^{\mathrm{h}, \mathrm{v}}=\frac{7579}{110592000} h^{10}-\frac{622709811499}{1070176665600000} h^{12}
$$$$
t_{3,2}^{\mathrm{h}, \mathrm{h}}=\frac{7579}{110592000} h^{10}-\frac{622709811499}{1070176665600000} h^{12}
$$$$
t_{\frac{7}{2}, \frac{3}{2}}^{\mathrm{h}, \mathrm{v}}=\frac{7579}{165888000} h^{10}-\frac{1225804705393}{3210529996800000} h^{12}
$$ 


$$
\begin{aligned}
t_{4,1}^{\mathrm{h}, \mathrm{h}} & =\frac{7579}{165888000} h^{10}-\frac{975039706867}{3210529996800000} h^{12} \\
t_{\frac{9}{2}, \frac{1}{2}}^{\mathrm{h}, \mathrm{v}} & =\frac{7579}{663552000} h^{10}-\frac{85039213657}{917294284800000} h^{12} \\
t_{5,0}^{\mathrm{h}, \mathrm{h}} & =\frac{7579}{663552000} h^{10}-\frac{11672061361}{229323571200000} h^{12} \\
t_{0,6}^{\mathrm{h}, \mathrm{h}} & =0 \\
t_{\frac{1}{2}, \frac{11}{2}}^{\mathrm{h}, \mathrm{v}} & =-\frac{168493133}{91729428480000} h^{12} \\
t_{1,5}^{\mathrm{h}, \mathrm{h}} & =-\frac{168493133}{91729428480000} h^{12} \\
t_{\frac{3}{2}, \frac{9}{2}}^{\mathrm{h}, \mathrm{v}} & =-\frac{168493133}{18345885696000} h^{12} \\
t_{2,4}^{\mathrm{h}, \mathrm{h}} & =-\frac{168493133}{18345885696000} h^{12} \\
t_{\frac{5}{2}, \frac{7}{2}}^{\mathrm{h}, \mathrm{v}} & =-\frac{168493133}{9172942848000} h^{12} \\
t_{3,3}^{\mathrm{h}, \mathrm{h}} & =-\frac{168493133}{9172942848000} h^{12} \\
t_{\frac{7}{2}, \frac{5}{2}}^{\mathrm{h}, \mathrm{v}} & =-\frac{168493133}{9172942848000} h^{12} \\
t_{4, \mathrm{~h}}^{\mathrm{h}, \mathrm{h}} & =-\frac{168493133}{9172942848000} h^{12} \\
t_{\frac{9}{2}, \frac{3}{2}}^{\mathrm{h}, \mathrm{v}} & =-\frac{168493133}{18345885696000} h^{12} \\
t_{5,1}^{\mathrm{h}, \mathrm{h}} & =-\frac{168493133}{18345885696000} h^{12} \\
t_{\frac{11}{2}, \frac{1}{2}}^{\mathrm{h}, \mathrm{v}} & =-\frac{168493133}{91729428480000} h^{12} \\
t_{6,0}^{\mathrm{h}, \mathrm{h}} & =-\frac{168493133}{91729428480000} h^{12} \\
& =-10
\end{aligned}
$$

\section{Appendix B: Low-energy spectrum of the XXZ}

Notations are the same as in Appendix A.

\section{One-magnon hopping amplitudes}

$$
\begin{aligned}
t_{0,0}= & 4-\frac{1}{3} \lambda^{2}+\frac{287}{864} \lambda^{4}-\frac{910529}{6220800} \lambda^{6} \\
& +\frac{5792068288969}{57610828800000} \lambda^{8} \\
t_{1,1}= & -\frac{1}{2} \lambda^{2}+\frac{11}{72} \lambda^{4}-\frac{2106349}{31104000} \lambda^{6} \\
& +\frac{19716698831861}{307257753600000} \lambda^{8} \\
t_{2,0}= & -\frac{1}{4} \lambda^{2}+\frac{1}{72} \lambda^{4}-\frac{752221}{20736000} \lambda^{6} \\
& +\frac{19657769838433}{614515507200000} \lambda^{8} \\
t_{2,2}= & -\frac{7}{192} \lambda^{4}-\frac{279853}{31104000} \lambda^{6}+\frac{1163045360221}{153628876800000} \lambda^{8}
\end{aligned}
$$

$$
\begin{aligned}
t_{4,0} & =-\frac{7}{1152} \lambda^{4}-\frac{578213}{62208000} \lambda^{6}+\frac{14632795561}{5486745600000} \lambda^{8} \\
t_{3,3} & =-\frac{251}{27648} \lambda^{6}+\frac{2563786289}{10241925120000} \lambda^{8} \\
t_{4,2} & =-\frac{251}{36864} \lambda^{6}-\frac{17610038713}{20483850240000} \lambda^{8} \\
t_{5,1} & =-\frac{251}{92160} \lambda^{6}-\frac{39527719681}{20483850240000} \lambda^{8} \\
t_{6,0} & =-\frac{251}{552960} \lambda^{6}-\frac{3452274997}{2926264320000} \lambda^{8} \\
t_{4,4} & =-\frac{327349}{159252480} \lambda^{8} \\
t_{5,3} & =-\frac{327349}{199065600} \lambda^{8} \\
t_{6,2} & =-\frac{327349}{398131200} \lambda^{8} \\
t_{7,1} & =-\frac{327349}{1393459200} \lambda^{8} \\
t_{8,0} & =-\frac{327349}{11147673600} \lambda^{8}
\end{aligned}
$$

2. Two-magnon bound state hopping amplitudes

$$
\begin{aligned}
& t_{0,0}^{\mathrm{h}, \mathrm{h}}=6-\frac{1}{2} \lambda^{2}-\frac{229}{2880} \lambda^{4}-\frac{10916263}{12441600} \lambda^{6} \\
& +\frac{77090072016313}{76814438400000} \lambda^{8} \\
& t_{0,1}^{\mathrm{h}, \mathrm{h}}=-\frac{1}{3} \lambda^{2}+\frac{67}{216} \lambda^{4}-\frac{4985783}{24883200} \lambda^{6} \\
& +\frac{231067152851203}{263363788800000} \lambda^{8} \\
& t_{\frac{1}{2}, \frac{1}{2}}^{\mathrm{h}, \mathrm{v}}=-\frac{1}{3} \lambda^{2}+\frac{89}{432} \lambda^{4}-\frac{943667}{2764800} \lambda^{6} \\
& +\frac{223581725823587}{263363788800000} \lambda^{8} \\
& t_{1,0}^{\mathrm{h}, \mathrm{h}}=\frac{1}{6} \lambda^{2}+\frac{11}{288} \lambda^{4}-\frac{584821}{6220800} \lambda^{6} \\
& +\frac{10646999914133}{26336378880000} \lambda^{8} \\
& t_{0,2}^{\mathrm{h}, \mathrm{h}}=-\frac{257}{1728} \lambda^{4}-\frac{123863}{921600} \lambda^{6}+\frac{14069731116209}{184354652160000} \lambda^{8} \\
& t_{\frac{1}{2}, \frac{3}{2}}^{\mathrm{h}, \mathrm{v}}=-\frac{1}{12} \lambda^{2}-\frac{29}{864} \lambda^{4}-\frac{2229119}{49766400} \lambda^{6} \\
& +\frac{4078695604273}{15049359360000} \lambda^{8} \\
& t_{1,1}^{\mathrm{h}, \mathrm{h}}=-\frac{1}{12} \lambda^{2}-\frac{41}{576} \lambda^{4}-\frac{335161}{2488320} \lambda^{6} \\
& +\frac{3645776906291}{17557585920000} \lambda^{8} \\
& t_{\frac{3}{2}, \frac{1}{2}}^{\mathrm{h}, \mathrm{v}}=-\frac{1}{12} \lambda^{2}-\frac{29}{864} \lambda^{4}-\frac{2229119}{49766400} \lambda^{6} \\
& +\frac{4078695604273}{15049359360000} \lambda^{8}
\end{aligned}
$$




$$
\begin{aligned}
& t_{2,0}^{\mathrm{h}, \mathrm{h}}=-\frac{1}{12} \lambda^{2}-\frac{11}{432} \lambda^{4}-\frac{857713}{12441600} \lambda^{6} \\
& +\frac{688142204467}{9405849600000} \lambda^{8} \\
& t_{0,3}^{\mathrm{h}, \mathrm{h}}=-\frac{1}{1728} \lambda^{4}+\frac{970931}{62208000} \lambda^{6}+\frac{23063997226177}{921773260800000} \lambda^{8} \\
& t_{\frac{1}{2}, \frac{5}{2}}^{\mathrm{h}, \mathrm{v}}=\frac{1}{432} \lambda^{4}-\frac{244283}{41472000} \lambda^{6}+\frac{30735370205093}{921773260800000} \lambda^{8} \\
& t_{1,2}^{\mathrm{h}, \mathrm{h}}=\frac{7}{1728} \lambda^{4}-\frac{12331}{15552000} \lambda^{6}+\frac{207639631734467}{1843546521600000} \lambda^{8} \\
& t_{\frac{3}{2}, \frac{3}{2}}^{\mathrm{h}, \mathrm{v}}=\frac{1}{192} \lambda^{4}-\frac{270403}{4976640} \lambda^{6}+\frac{26215520575403}{307257753600000} \lambda^{8} \\
& t_{2,1}^{\mathrm{h}, \mathrm{h}}=\frac{1}{192} \lambda^{4}-\frac{428399}{24883200} \lambda^{6}+\frac{14013035309131}{184354652160000} \lambda^{8} \\
& t_{\frac{5}{2}, \frac{1}{2}}^{\mathrm{h}, \mathrm{v}}=\frac{1}{432} \lambda^{4}-\frac{244283}{41472000} \lambda^{6}+\frac{30735370205093}{921773260800000} \lambda^{8} \\
& t_{3,0}^{\mathrm{h}, \mathrm{h}}=\frac{1}{1728} \lambda^{4}-\frac{7861}{6220800} \lambda^{6}+\frac{282505209937}{46088663040000} \lambda^{8} \\
& t_{0,4}^{\mathrm{h}, \mathrm{h}}=-\frac{7277}{12441600} \lambda^{6}+\frac{28585558937}{307257753600000} \lambda^{8} \\
& t_{\frac{1}{2}, \frac{7}{2}}^{\mathrm{h}, \mathrm{v}}=-\frac{1}{3456} \lambda^{4}+\frac{298777}{248832000} \lambda^{6}+\frac{1744637644499}{245806202880000} \lambda^{8} \\
& t_{1,3}^{\mathrm{h}, \mathrm{h}}=-\frac{1}{3456} \lambda^{4}+\frac{77}{124416} \lambda^{6}-\frac{4258929380371}{921773260800000} \lambda^{8} \\
& t_{\frac{3}{2}, \frac{5}{2}}^{\mathrm{h}, \mathrm{v}}=-\frac{1}{1152} \lambda^{4}+\frac{406687}{248832000} \lambda^{6}+\frac{1166929622801}{73741860864000} \lambda^{8} \\
& t_{2,2}^{\mathrm{h}, \mathrm{h}}=-\frac{1}{1152} \lambda^{4}+\frac{9367}{2488320} \lambda^{6}+\frac{31971087871}{7681443840000} \lambda^{8} \\
& t_{\frac{5}{2}, \frac{3}{2}}^{\mathrm{h}, \mathrm{v}}=-\frac{1}{1152} \lambda^{4}+\frac{406687}{248832000} \lambda^{6}+\frac{1166929622801}{73741860864000} \lambda^{8} \\
& t_{3,1}^{\mathrm{h}, \mathrm{h}}=-\frac{1}{1152} \lambda^{4}+\frac{14579}{6912000} \lambda^{6}+\frac{18122969387783}{1843546521600000} \lambda^{8} \\
& t_{\frac{7}{2}, \frac{1}{2}}^{\mathrm{h}, \mathrm{v}}=-\frac{1}{3456} \lambda^{4}+\frac{298777}{248832000} \lambda^{6}+\frac{1744637644499}{245806202880000} \lambda^{8} \\
& t_{4,0}^{\mathrm{h}, \mathrm{h}}=-\frac{1}{3456} \lambda^{4}-\frac{15667}{31104000} \lambda^{6}+\frac{1013784889699}{307257753600000} \lambda^{8} \\
& t_{0,5}^{\mathrm{h}, \mathrm{h}}=-\frac{217}{12441600} \lambda^{6}-\frac{12986330681}{92177326080000} \lambda^{8} \\
& t_{\frac{1}{2}, \frac{9}{2}}^{\mathrm{h}, \mathrm{v}}=-\frac{991}{6220800} \lambda^{6}-\frac{1247791850591}{1843546521600000} \lambda^{8} \\
& t_{1,4}^{\mathrm{h}, \mathrm{h}}=-\frac{4181}{12441600} \lambda^{6}-\frac{2538082499891}{921773260800000} \lambda^{8} \\
& t_{\frac{3}{2}, \frac{7}{2}}^{\mathrm{h}, \mathrm{v}}=-\frac{120691}{124416000} \lambda^{6}-\frac{99157135381}{3687093043200000} \lambda^{8} \\
& t_{2,3}^{\mathrm{h}, \mathrm{h}}=-\frac{20177}{15552000} \lambda^{6}-\frac{26346818453}{34139750400000} \lambda^{8} \\
& t_{\frac{5}{2}, \frac{5}{2}}^{\mathrm{h}, \mathrm{v}}=-\frac{25489}{15552000} \lambda^{6}+\frac{1118638298843}{921773260800000} \lambda^{8} \\
& t_{3,2}^{\mathrm{h}, \mathrm{h}}=-\frac{25489}{15552000} \lambda^{6}+\frac{415281490573}{153628876800000} \lambda^{8} \\
& t_{\frac{7}{2}, \frac{3}{2}}^{\mathrm{h}, \mathrm{v}}=-\frac{120691}{124416000} \lambda^{6}-\frac{99157135381}{3687093043200000} \lambda^{8} \\
& t_{4,1}^{\mathrm{h}, \mathrm{h}}=-\frac{39983}{62208000} \lambda^{6}+\frac{690474135503}{921773260800000} \lambda^{8} \\
& t_{\frac{9}{2}, \frac{1}{2}}^{\mathrm{h}, \mathrm{v}}=-\frac{991}{6220800} \lambda^{6}-\frac{1247791850591}{1843546521600000} \lambda^{8}
\end{aligned}
$$

$$
\begin{aligned}
t_{5,0}^{\mathrm{h}, \mathrm{h}} & =\frac{217}{12441600} \lambda^{6}-\frac{17201780639}{184354652160000} \lambda^{8} \\
t_{0,6}^{\mathrm{h}, \mathrm{h}} & =\frac{11448487}{1755758592000} \lambda^{8}
\end{aligned}
$$$$
t_{\frac{1}{2}, \frac{11}{2}}^{\mathrm{h}, \mathrm{v}}=-\frac{217}{24883200} \lambda^{6}-\frac{32024937673}{737418608640000} \lambda^{8}
$$$$
t_{1,5}^{\mathrm{h}, \mathrm{h}}=-\frac{217}{24883200} \lambda^{6}-\frac{12649730881}{368709304320000} \lambda^{8}
$$$$
t_{\frac{3}{2}, \frac{9}{2}}^{\mathrm{h}, \mathrm{v}}=-\frac{217}{4976640} \lambda^{6}-\frac{158928910813}{737418608640000} \lambda^{8}
$$$$
t_{2,4}^{\mathrm{h}, \mathrm{h}}=-\frac{217}{4976640} \lambda^{6}-\frac{10506217709}{92177326080000} \lambda^{8}
$$$$
t_{\frac{5}{2}, \frac{7}{2}}^{\mathrm{h}, \mathrm{v}}=-\frac{217}{2488320} \lambda^{6}-\frac{154367383127}{368709304320000} \lambda^{8}
$$$$
t_{3,3}^{\mathrm{h}, \mathrm{h}}=-\frac{217}{2488320} \lambda^{6}-\frac{7497652279}{20483850240000} \lambda^{8}
$$$$
t_{\frac{7}{2}, \frac{5}{2}}^{\mathrm{h}, \mathrm{v}}=-\frac{217}{2488320} \lambda^{6}-\frac{154367383127}{368709304320000} \lambda^{8}
$$$$
t_{4,2}^{\mathrm{h}, \mathrm{h}}=-\frac{217}{2488320} \lambda^{6}-\frac{104001673121}{184354652160000} \lambda^{8}
$$$$
t_{\frac{9}{2}, \frac{3}{2}}^{\mathrm{h}, \mathrm{v}}=-\frac{217}{4976640} \lambda^{6}-\frac{158928910813}{737418608640000} \lambda^{8}
$$$$
t_{5,1}^{\mathrm{h}, \mathrm{h}}=-\frac{217}{4976640} \lambda^{6}-\frac{101559204737}{368709304320000} \lambda^{8}
$$$$
t_{\frac{11}{2}, \frac{1}{2}}^{\mathrm{h}, \mathrm{v}}=-\frac{217}{24883200} \lambda^{6}-\frac{32024937673}{737418608640000} \lambda^{8}
$$$$
t_{6,0}^{\mathrm{h}, \mathrm{h}}=-\frac{217}{24883200} \lambda^{6}-\frac{5743189}{1280240640000} \lambda^{8}
$$$$
t_{0,7}^{\mathrm{h}, \mathrm{h}}=-\frac{421}{5573836800} \lambda^{8}
$$$$
t_{\frac{1}{2}, \frac{13}{2}}^{\mathrm{h}, \mathrm{v}}=\frac{2696353}{1755758592000} \lambda^{8}
$$$$
t_{1,6}^{\mathrm{h}, \mathrm{h}}=\frac{5260091}{1755758592000} \lambda^{8}
$$$$
t_{\frac{3}{2}, \frac{11}{2}}^{\mathrm{h}, \mathrm{v}}=\frac{15978763}{548674560000} \lambda^{8}
$$$$
t_{2,5}^{\mathrm{h}, \mathrm{h}}=\frac{103416781}{2926264320000} \lambda^{8}
$$$$
t_{\frac{5}{2}, \frac{9}{2}}^{\mathrm{h}, \mathrm{v}}=\frac{896000347}{8778792960000} \lambda^{8}
$$$$
t_{3,4}^{\mathrm{h}, \mathrm{h}}=\frac{965398397}{8778792960000} \lambda^{8}
$$$$
t_{\frac{7}{2}, \frac{7}{2}}^{\mathrm{h}, \mathrm{v}}=\frac{1308306883}{8778792960000} \lambda^{8}
$$$$
t_{4,3}^{\mathrm{h}, \mathrm{h}}=\frac{1308306883}{8778792960000} \lambda^{8}
$$$$
t_{\frac{9}{2}, \frac{5}{2}}^{\mathrm{h}, \mathrm{v}}=\frac{896000347}{8778792960000} \lambda^{8}
$$$$
t_{5,2}^{\mathrm{h}, \mathrm{h}}=\frac{275534099}{2926264320000} \lambda^{8}
$$$$
t_{\frac{11}{2}, \frac{3}{2}}^{\mathrm{h}, \mathrm{v}}=\frac{15978763}{548674560000} \lambda^{8}
$$$$
t_{6,1}^{\mathrm{h}, \mathrm{h}}=\frac{201070073}{8778792960000} \lambda^{8}
$$$$
t_{\frac{13}{2}, \frac{1}{2}}^{\mathrm{h}, \mathrm{v}}=\frac{2696353}{1755758592000} \lambda^{8}
$$ 


$$
\begin{aligned}
t_{7,0}^{\mathrm{h}, \mathrm{h}} & =\frac{421}{5573836800} \lambda^{8} \\
t_{0,8}^{\mathrm{h}, \mathrm{h}} & =0 \\
t_{\frac{1}{2}, \frac{15}{2}}^{\mathrm{h}, \mathrm{v}} & =-\frac{421}{11147673600} \lambda^{8} \\
t_{1,7}^{\mathrm{h}, \mathrm{h}} & =-\frac{421}{11147673600} \lambda^{8} \\
t_{\frac{3}{2}, \frac{13}{2}}^{\mathrm{h}, \mathrm{v}} & =-\frac{421}{1592524800} \lambda^{8} \\
t_{2,6}^{\mathrm{h}, \mathrm{h}} & =-\frac{421}{1592524800} \lambda^{8} \\
t_{\frac{5}{2}, \frac{11}{2}}^{\mathrm{h}, \mathrm{v}} & =-\frac{421}{530841600} \lambda^{8} \\
t_{3,5}^{\mathrm{h}, \mathrm{h}} & =-\frac{421}{530841600} \lambda^{8} \\
t_{\frac{7}{2}, \frac{9}{2}}^{\mathrm{h}, \mathrm{v}} & =-\frac{421}{318504960} \lambda^{8} \\
t_{4,4}^{\mathrm{h}, \mathrm{h}} & =-\frac{421}{318504960} \lambda^{8} \\
t_{\frac{9}{2}, \frac{7}{2}}^{\mathrm{h}, \mathrm{v}} & =-\frac{421}{318504960} \lambda^{8} \\
t_{5,3}^{\mathrm{h}, \mathrm{h}} & =-\frac{421}{318504960} \lambda^{8} \\
t_{\frac{11}{2}, \frac{5}{2}}^{\mathrm{h}, \mathrm{v}} & =-\frac{421}{530841600} \lambda^{8} \\
t_{6,2}^{\mathrm{h}, \mathrm{h}} & =-\frac{421}{530841600} \lambda^{8} \\
t_{\frac{13}{2}, \frac{3}{2}}^{\mathrm{h}, \mathrm{v}} & =-\frac{421}{1592524800} \lambda^{8} \\
t_{7,1}^{\mathrm{h}, \mathrm{h}} & =-\frac{421}{1592524800} \lambda^{8} \\
t_{\frac{15}{2}, \frac{1}{2}}^{\mathrm{h}, \mathrm{v}} & =-\frac{421}{11147673600} \lambda^{8} \\
t_{8,0}^{\mathrm{h}, \mathrm{h}} & =-\frac{421}{11147673600} \lambda^{8}
\end{aligned}
$$

* Electronic address: sdusuel@gmail.com

† Electronic address: kamfor@fkt.physik.uni-dortmund.de

¥ Electronic address: schmidt@fkt.physik.uni-dortmund.de

$\S$ Electronic address: rthomale@princeton.edu

ฯ Electronic address: vidal@lptmc.jussieu.fr

1 S. Sachdev, Quantum Phases Transitions (Cambridge University Press, Cambridge, 1999).

2 B. Douçot and J. Vidal, Phys. Rev. Lett. 88, 227005 (2002).

3 J. Vidal, B. Douçot, R. Mosseri, and P. Butaud, Phys. Rev. Lett. 85, 3906 (2000).

4 S. Capponi, G. Roux, P. Azaria, E. Boulat, and P. Lecheminant, Phys. Rev. B 75, 100503(R) (2007).

${ }^{5}$ G. S. Uhrig and H. J. Schulz, Phys. Rev. B 54, R9624 (1996).

6 S. Trebst, H. Monien, C. J. Hamer, W. H. Zheng, and R. R. P. Singh, Phys. Rev. Lett. 85, 4373 (2000).

7 W. H. Zheng, J. Oitmaa, and C. J. Hamer, Phys. Rev. B 43, 8321 (1991).
8 C. Knetter, K. P. Schmidt, M. Grüninger, and G. S. Uhrig, Phys. Rev. Lett. 87, 167204 (2001).

${ }^{9}$ W. H. Zheng, C. J. Hamer, R. R. P. Singh, S. Trebst, and H. Monien, Phys. Rev. B 63, 144410 (2001).

10 K. P. Schmidt, C. Knetter, and G. S. Uhrig, Phys. Rev. B 69, 104417 (2004).

11 M. Windt et al., Phys. Rev. Lett. 87, 127002 (2001).

12 C. Knetter, A. Bühler, E. Müller-Hartmann, and G. S. Uhrig, Phys. Rev. Lett. 85, 3958 (2000).

13 P. Lemmens, M. Grove, M. Fischer, G. Güntherodt, V. N. Kotov, H. Kageyama, K. Onizuka, and Y. Ueda, Phys. Rev. Lett. 85, 2605 (2000).

14 J. Vidal, R. Thomale, K. P. Schmidt, and S. Dusuel, Phys. Rev. B 80, 081104(R) (2009).

15 C. J. Hamer, Phys. Rev. B 79, 212413 (2009).

16 M. Caselle, M. Hasenbuch, and P. Provero, Nucl. Phys. B 556, 575 (1999).

17 M. Caselle, M. Hasenbuch, P. Provero, and K. Zarembo, Nucl. Phys. B 623, 474 (2002). 
18 M. P. Gelfand and R. R. P. Singh, Adv. Phys. 49, 93 (2000).

19 J. Oitmaa, C. J. Hamer, and W. H. Zheng, Series Expansion Methods for Strongly Interacting Lattice Models (Cambridge University Press, Cambridge, 2006).

${ }^{20}$ F. Wegner, Ann. Phys. (Leipzig) 3, 77 (1994).

21 J. Stein, J. Stat. Phys. 88, 487 (1997).

22 C. Knetter and G. S. Uhrig, Eur. Phys. J. B 13, 209 (2000).

23 C. Knetter, K. P. Schmidt, and G. S. Uhrig, J. Phys. A 36, 7889 (2003).

24 I. G. Enting, Nucl. Phys. B (Proc. Suppl.) 47, 180 (1996).

25 A. Y. Kitaev, Ann. Phys. (N.Y.) 303, 2 (2003).

26 J. Vidal, S. Dusuel, and K. P. Schmidt, Phys. Rev. B 79, 033109 (2009).

27 H.-X. He, C. J. Hamer, and J. Oitmaa, J. Phys. A 23, 1775 (1990).

28 J. Oitmaa, C. J. Hamer, and W. H. Zheng, J. Phys. A 24, 2863 (1991).

29 R. R. P. Singh, Phys. Rev. B 39, 9760 (1989).

30 W. H. Zheng, C. J. Hamer, and R. R. P. Singh, Phys. Rev. Lett. 91, 037206 (2003).

31 J. Oitmaa, C. J. Hamer, and W. H. Zheng, Phys. Rev. B 50, 3877 (1994).

32 S. Trebst, P. Werner, M. Troyer, K. Shtengel, and C. Nayak, Phys. Rev. Lett. 98, 070602 (2007).

33 A. Hamma and D. A. Lidar, Phys. Rev. Lett. 100, 030502 (2008).

${ }^{34}$ I. S. Tupitsyn, A. Kitaev, N. V. Prokof'ev, and P. C. E. Stamp, arXiv:0804.3175.

35 E. Cobanera, G. Ortiz, and Z. Nussinov, Phys. Rev. Lett. 104, 020402 (2010).

36 J. Vidal, K. P. Schmidt, and S. Dusuel, Phys. Rev. B 78, 245121 (2008).
37 S. D. Głazek and K. G. Wilson, Phys. Rev. D 48, 5863 (1993).

38 S. D. Głazek and K. G. Wilson, Phys. Rev. D 49, 4214 (1994).

39 S. Dusuel and G. S. Uhrig, J. Phys. A 37, 9275 (2004).

40 S. Dusuel and J. Vidal, Phys. Rev. B 71, 224420 (2005).

41 H. Primas, Rev. Mod. Phys. 35, 710 (1963).

42 I. Shavitt and L. T. Redmon, J. Chem. Phys. 73, 5711 (1980).

43 T. de Neef and I. G. Enting, J. Phys. A 10, 801 (1977).

${ }^{44}$ H. Arisue and T. Fujiwara, Phys. Rev. E 67, 066109 (2003).

45 R. Thomale, E. Kapit, D. F. Schroeter, and M. Greiter, Phys. Rev. B 80, 104406 (2009).

46 H. Arisue, T. Fujiwara, and K. Tabata, Nucl. Phys. B (Proc. Suppl.) 129-130, 774 (2004).

47 Y. Nishiyama, Phys. Rev. E 77, 051112 (2008).

48 T. Fischer, S. Duffe, and G. S. Uhrig, arXiv:0911.5007.

49 R. R. P. Singh, P. A. Fleury, K. B. Lyons, and P. E. Sulewski, Phys. Rev. Lett. 62, 2736 (1989).

50 C. M. Canali and S. M. Girvin, Phys. Rev. B 45, 7127 (1992).

${ }^{51}$ K. B. Lyons, P. E. Sulewski, P. A. Fleury, H. L. Carter, A. S. Cooper, G. P. Espinosa, Z. Fisk, and S.-W. Cheong, Phys. Rev. B 39, 9693 (1989).

52 I. Ohana, M. S. Dresselhaus, Y. C. Liu, P. J. Picone, D. R. Gabbe, H. P. Jenssen, and G. Dresselhaus, Phys. Rev. B 39, 2293 (1989).

53 C. Xu and J. E. Moore, Phys. Rev. Lett. 93, 047003 (2004).

54 F. Wegner, J. Math. Phys. 12, 2259 (1971).

55 T. Oguchi and T. Ishikawa, J. Phys. Soc. Jpn. 34, 1486 (1973). 